\title{
Estimating the level of dynamical noise in time series by using fractal dimensions
}

\author{
Takumi Sase ${ }^{\mathrm{a}, *}$, Jonatán Peña Ramírez ${ }^{\mathrm{b}}$, Keiichi Kitajo ${ }^{\mathrm{c}}$, Kazuyuki Aihara $^{\mathrm{a}, \mathrm{d}}$, \\ Yoshito Hirata ${ }^{\mathrm{a}, \mathrm{d}}$ \\ ${ }^{a}$ Graduate School of Information Science and Technology, The University of Tokyo, Tokyo \\ 153-8505, Japan \\ ${ }^{b}$ CONACYT Research Fellow, Center for Scientic Research and Higher Education at \\ Ensenada (CICESE), Carretera Ensenada-Tijuana No. 3918, Zona Playitas, C.P. 22860, \\ Ensenada, Baja California, Mexico \\ ${ }^{c}$ Rhythm-based Brain Information Processing Unit, BSI-Toyota Collaboration Center, \\ RIKEN Brain Science Institute, Wako, Saitama 351-0198, Japan \\ ${ }^{d}$ Institute of Industrial Science, The University of Tokyo, Tokyo 153-8505, Japan
}

\begin{abstract}
We present a method for estimating the dynamical noise level of a 'short' time series even if the dynamical system is unknown. The proposed method estimates the level of dynamical noise by calculating the fractal dimensions of the time series. Additionally, the method is applied to EEG data to demonstrate its possible effectiveness as an indicator of temporal changes in the level of dynamical noise.

Key words: Dynamical noise level, Fractal dimension, Short time series, EEG data

2010 MSC: 00-01, 99-00
\end{abstract}

\section{Introduction}

In general, time series data-for example, biological, meteorological, and economic data - are a collection of measurements recording the dynamical behavior of a system. When the underlying dynamics of the system is stochastic, the associated time series will behave like a stochastic process. Note, however,

\footnotetext{
* Corresponding author

Email addresses: sase@sat.t.u-tokyo.ac.jp (Takumi Sase)
}

Preprint submitted to Physics Letters A

January 7, 2016

(C) 2016. This manuscript version is made available under the Elsevier user license http://www.elsevier.com/open-access/userlicense/1.0/ 
that the time series generated from deterministic systems may also be associated with stochastic fluctuations if the measured signals have been influenced by noise.

Typically, a time series contains two basic types of noise: dynamical noise and observational noise. The former refers to the noise affecting the system's dynamics, whereas the latter describes the noise influencing the observed or measured signal of the system. The system including these two distinctive kinds of noise is defined as follows [1]:

$$
\begin{aligned}
\mathrm{d} x_{q} & =f_{q}(\vec{x}) \mathrm{d} t+\rho_{q}(t) \mathrm{d} W_{q}(t), \text { for } q=1,2, \ldots, d, \\
X_{\mathrm{obs}}(t) & =H(\vec{x})+\sigma_{\mathrm{X}} \xi_{\mathrm{X}}(t),
\end{aligned}
$$

where $x_{q}$ denotes the state variable, $\vec{x}=\left[x_{1}, x_{2}, \ldots, x_{d}\right]^{\mathrm{T}}$ is the state vector, function $f_{q}(\cdot)$ is either linear or nonlinear, and the dynamical noise is described by $\rho_{q}(t) \mathrm{d} W_{q}(t)$. Here, $\mathrm{d} W_{q}(t)$ indicates the Gaussian white noise, which follows a normal distribution with mean 0 and standard deviation $\sqrt{\mathrm{d} t}$. Note that, in order to define the dynamical noise as colored noise, the process of colored noise generated by the Gaussian white noise should be included in $f_{q}(\vec{x})$ so that the definition of a system [Eq. (1)] works more generally in terms of modeling the dynamical noise. We explicitly assume that the temporal change of $\rho_{q}(t)$ is slow compared with the dynamics of $x_{q}$. Note that Eq. (1) denotes a $d$-dimensional stochastic dynamical system, and the corresponding observational process of this system is described by Eq. (2). Furthermore, from Eq. (1) and Eq. (2), it can be seen that $\vec{x}$ is converted to a one-dimensional variable $X_{\text {obs }}(t)$ through an observational function $H(\cdot)$ with observational noise $\sigma_{\mathrm{X}} \xi_{\mathrm{X}}(t)$, where $\xi_{\mathrm{X}}(t)$ follows a normal distribution with mean 0 and standard deviation 1 . Usually, the corresponding time series of $\left\{X_{\mathrm{obs}}(t)\right\}$ is defined as follows:

$$
\{X(i)\}=\left\{X_{\mathrm{obs}}(t) \mid X_{\mathrm{obs}}(t)=X_{\mathrm{obs}}(i \Delta t), i \in \mathbb{N}\right\}
$$

where $\left\{X_{\mathrm{obs}}(t)\right\}$ is converted to a time series $\{X(i)\}$ with sampling time $\Delta t$.

In this study, we assume that the amount of dynamical noise can be described as a time-dependent variable, which is denoted by $\rho_{q}(t)$ in Eq. (1). 
For example, consider a situation in which the amount of noise in a neural system is temporally adjusted by input from other areas of the brain to improve brain functions. The amount of observational noise, denoted by $\sigma_{\mathrm{X}}$ in Eq. (2), is assumed to be constant. This assumption is equivalent to the assumption that the measurement environment does not change during the observational process. For a measured signal of the form $X_{\mathrm{obs}}(t)=x_{q}(t)+\sigma_{\mathrm{X}} \xi_{\mathrm{X}}(t)$, the dynamical and observational noise levels have been defined as $\rho_{q}(t) / \sigma_{\operatorname{dyn} x_{q}}(t)$ and $\sigma_{\mathrm{X}} / \sigma_{\mathrm{dyn} x_{q}}(t)$, respectively, where $\sigma_{\operatorname{dyn} x_{q}}(t)$ indicates the standard deviation of the variable $x_{q}$. The time dependency of $\sigma_{\operatorname{dyn} x_{q}}(t)$ can be attributed to the fact that, in general, the dynamical system [Eq. (1)] behaves as a nonstationary process.

From the system's dynamics of Eq. (1), it is clear that dynamical noise may influence the behavior of the dynamical system. In fact, a large dynamical noise level increases the probability of a large trajectory change. The steady state of the dynamical system may drift into different regions in the state space. Thus, knowing the dynamical noise level is a fundamental to analyzing and understanding dynamical systems $[2,3]$.

However, dynamical noise is more difficult to be estimated than observational noise because the former is directly added to the dynamics of time evolution. If the dynamical system is known, we can formulate algorithms for estimating the dynamical noise level $[4,5]$. Nevertheless, it should be noted that actual systems in the real world are usually unknown. For these systems, few methods have been proposed to estimate the dynamical noise level. For example, according to the work of Siefert et al. [6], an interesting quantitative method for analyzing dynamical noise has been proposed. This method can estimate the amount of noise in systems with unknown dynamics by using the KramersMoyal coefficients $[7,8]$, but the method requires a 'long' time series to estimate the mean and variance of a trajectory moving on an attractor. On the other hand, Urbanowicz and Holyst [9] proposed an important noise-level estimation method that assumes observational noise. Although they have suggested that their method is valid for observational noise as well as dynamical noise, we have 
found that their algorithm does not necessarily work well for estimation of the dynamical noise level.

In this paper, we propose a method for estimating the level of dynamical noise, which can be applied to even 'short' time series generated from unknown dynamics. This paper is organized as follows. First, in Section 2, we present some definitions that are useful for the subsequent results. Next, in Section 3, we recall Urbanowicz and Holyst's method [9], with the numerical verification whether their method is applicable for even short time series. Then, in Section 4, we introduce the proposed method and verify its effectiveness as a dynamical noise indicator. Furthermore, in Section 5, our method is applied to human electroencephalographic (EEG) data in order to illustrate its applicability to real-world systems. Finally, discussions of the obtained results are presented in Section 6, and some conclusions are provided in Section 7.

\section{Preliminaries}

We analytically derive some definitions for short and long time series as follows. First, we define

$$
\begin{aligned}
I_{1}(c) & =\int_{t_{0}}^{c} \frac{\left|\mathrm{E}_{\tau+h}\left[\left\{X_{\mathrm{obs}}(t)\right\}\right]-\mathrm{E}_{\tau}\left[\left\{X_{\mathrm{obs}}(t)\right\}\right]\right|}{h} \mathrm{~d} \tau \\
I_{2}(c) & =\int_{t_{0}}^{c} \frac{\left|\operatorname{Var}_{\tau+h}\left[\left\{X_{\mathrm{obs}}(t)\right\}\right]-\operatorname{Var}_{\tau}\left[\left\{X_{\mathrm{obs}}(t)\right\}\right]\right|}{h} \mathrm{~d} \tau,
\end{aligned}
$$

where $0<h \ll 1$, and operators $\mathrm{E}_{\tau}[\cdot]$ and $\operatorname{Var}_{\tau}[\cdot]$ denote the mean and variance, respectively, and are defined as follows:

$$
\begin{aligned}
\mathrm{E}_{\tau}\left[\left\{X_{\mathrm{obs}}(t)\right\}\right] & =\frac{1}{h^{\prime}} \int_{\tau}^{\tau+h^{\prime}} X_{\mathrm{obs}}(t) \mathrm{d} t \\
\operatorname{Var}_{\tau}\left[\left\{X_{\mathrm{obs}}(t)\right\}\right] & =\frac{1}{h^{\prime}} \int_{\tau}^{\tau+h^{\prime}}\left(X_{\mathrm{obs}}(t)-\mathrm{E}_{\tau}\left[\left\{X_{\mathrm{obs}}(t)\right\}\right]\right)^{2} \mathrm{~d} t
\end{aligned}
$$

with $0<h^{\prime} \ll 1$. The two quantities $I_{1}(c)$ and $I_{2}(c)$ indicate the sums of discrepancies $|\cdot|$ of 'partially' observational signals $\left\{X_{\mathrm{obs}}(s)\right\}$ over $\tau \leq s \leq \tau+h+h^{\prime}$ with $0 \leq t_{0} \leq c$ and $t_{0} \leq c \leq T$ [see the bidirectional arrows with width $h$ in Fig. 1(a)]; 
$t_{0}$ and $T$ are a starting point and an ending point of an observation, respectively. Then, if $\left\{X_{\mathrm{obs}}(s)\right\}$ behaves as a stationary process, both quantities $I_{1}(c)$ and $I_{2}(c)$ are almost equal to zero, even if $c$ becomes large, because the mean and variance of the stationary process do not change over time. However, in this study, we analyze signals observed from a stochastic dynamical system [see Eq. (1)], in which variables usually behave as nonstationary processes, so that observational signals also stand for nonstationarity. In fact, each of quantities $I_{1}(c)$ and $I_{2}(c)$ represents a margin between stationarity and nonstationarity [see Fig. 1]. Note that in Eq. (4) and Eq. (5), if $h$ is set to a very small value, the partial signal $\left\{X_{\mathrm{obs}}(s)\right\}$ can be considered as a 'semi-stationary' process.

Depending on whether the target signal is near stationarity, the present method works either as a universal indicator among systems or as an intrinsic indicator for each system. If the target signal is near stationarity, i.e., it is observed as a short time series, the method can universally extract the dynamical noise level for many systems; if the target signal is far from stationarity, i.e., it is observed as a long time series, the method can specifically extract the dynamical noise level for each system so that the extracted levels are not comparable among systems, but involve properties of their respective systems. Thus, it is essential to flexibly determine a boundary between short and long time series.

To choose a threshold value $c^{*}$, by which we can define whether a time series is short or long, we use Eq. (4) and Eq. (5) with two upper bounds $\epsilon_{1}\left(0<\epsilon_{1}\right)$ and $\epsilon_{2}\left(0<\epsilon_{2}\right)$, and compute $c_{1}^{*}$ and $c_{2}^{*}$ such that

$$
\begin{aligned}
& I_{1}\left(c_{1}^{*}\right)=\epsilon_{1} \\
& I_{2}\left(c_{2}^{*}\right)=\epsilon_{2} .
\end{aligned}
$$

${ }_{06}$ Then, we define the threshold value $c^{*}$ as follows:

$$
c^{*}=\min \left(c_{1}^{*}, c_{2}^{*}\right) \text {. }
$$

${ }_{107}$ The computation of $c^{*}$ is provided in Appendix A. This threshold value $c^{*}$ determines whether the length $L_{\text {seg }}\left(t_{0}, \Delta t\right)$ of a time series $\{X(i)\}$ is short or 

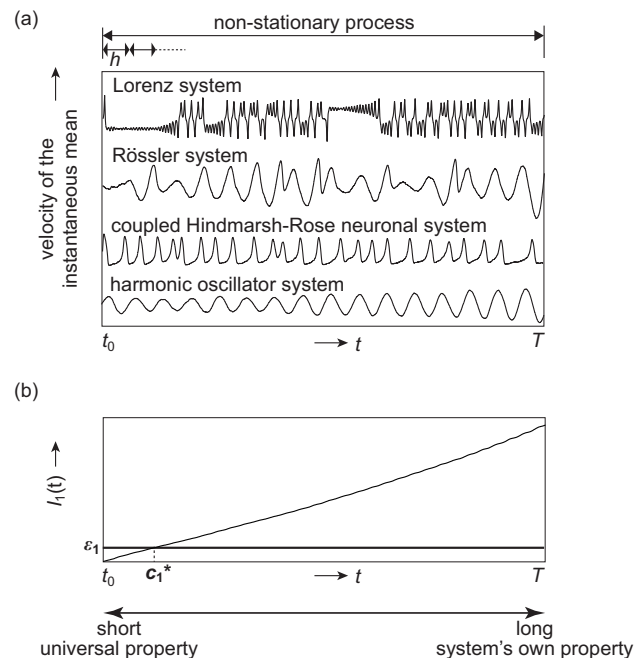

Figure 1: Concept of a separation between short and long time series by discrepancies introduced between stationarity and nonstationarity arising from the fact that a partially shortterm signal $X_{\text {obs }}(s)$ with very short length $h$ is a semi-stationary process. (a) Four time series sorted in accordance with the velocity of the instantaneous mean value of the time series from bottom to top: the harmonic oscillator system, the Rössler system, the coupled Hindmarsh-Rose neuronal system, and the Lorenz system. The time series were obtained with $\rho_{q}(t) / \sigma_{\operatorname{dyn} x_{q}}(t)=0.1$. (b) A schematic example of the accumulation $I_{1}(t)$ of discrepancies. An upper bound $\epsilon_{1}$ determines a threshold value $c_{1}^{*}$. If the accumulation $I_{1}(t)<\epsilon_{1}$, we call this time series a short time series, and $I_{1}(t)$ reflects a more universal property among systems. If $I_{1}(t)$ is large, we call this time series a long time series, and $I_{1}(t)$ reflects a more specific property of the system because the longer the time series increases, the more its wave form differs from those of other time series.

long. If the inequality

$$
L_{\text {seg }}\left(t_{0}, \Delta t\right) \leq\left(c^{*}-t_{0}\right) / \Delta t
$$

is satisfied, the time series is assumed to be short. Otherwise, the time series is referred to as a long time series. Note that Eq. (4) and Eq. (5) define $I_{1}\left(c_{1}^{*}\right)$ and $I_{2}\left(c_{2}^{*}\right)$ like differences, rather than derivatives because $\left\{X_{\mathrm{obs}}(t)\right\}$ may be a non-smooth time series. Equations (4)-(11) can be seen as a set of general definitions for the length of a time series generated from a continuous-time dynamical system. Throughout this paper, when we discuss whether a time 
series is short or long, we denote the length of the time series as $L_{\text {seg }}\left(t_{0}, \Delta t\right)$, otherwise as $N$, which refers to the data size of the time series.

\section{Previous method for noise-level estimation}

First, we introduce the previous method for noise-level estimation, which uses coarse-grained entropy [9]. Because this algorithm assumes observational noise, it may fail when trying to estimate the level of dynamical noise.

\subsection{Urbanowicz and Holyst's method}

Suppose that a time series $\{X(i)\}$ with $i=1,2, \ldots, N$ is given. Furthermore, assume that it is influenced neither by observational noise nor by dynamical noise, i.e. assume a 'clean' time series. Then, we construct $M$-dimensional delay coordinates as $\vec{X}_{M}(i)=\{X(i), X(i+\Gamma), \ldots, X(i+(M-1) \Gamma)$, where $M$ and $\Gamma$ are an embedding dimension and an embedding delay, respectively. We define the correlation integral with a threshold value $\varepsilon$ for $\vec{X}_{M}(i)$ as

$$
C^{M}(\varepsilon)=\frac{1}{N^{2}} \sum_{i}^{N} \sum_{p \neq i}^{N} \theta\left(\varepsilon-\left\|\vec{X}_{M}(i)-\vec{X}_{M}(p)\right\|\right),
$$

where $\theta(\cdot)$ is the Heaviside function, defined as

$$
\theta(u)=\left\{\begin{array}{cc}
0 & (u<0) \\
1 & (u \geq 0)
\end{array}\right.
$$

For simplicity, we use the maximum norm. In addition, the term $C^{M}(\varepsilon)$ allows to define the coarse-grained correlation entropy, denoted by $K_{2}(\varepsilon)$, as follows $[10,11]$ :

$$
K_{2}(\varepsilon)=\lim _{\varepsilon \rightarrow 0} \lim _{M \rightarrow \infty} \ln \frac{C^{M}(\varepsilon)}{C^{M+1}(\varepsilon)} \approx-\frac{\mathrm{d} \ln C^{M}(\varepsilon)}{\mathrm{d} M} .
$$

33 The term $K_{2}(\varepsilon)$ is an ideal entropy, which cannot be obtained in reality. In 34 practice, we obtain a noisy version of this entropy, i.e. $K_{\text {noisy }}(\varepsilon)[9]$ instead of $K_{2}(\varepsilon)$, when the Gaussian observational noise is added to $\{X(i)\}$; we call this 
$K_{\text {noisy }}(\varepsilon)$ as the observed coarse-grained entropy defined as follows:

$$
\begin{aligned}
K_{\text {noisy }}(\varepsilon)= & -\frac{1}{\Gamma} g\left(\frac{\varepsilon}{2 \sigma}\right) \ln \varepsilon+[\kappa+b \ln (1-\alpha \varepsilon)] \\
& \times\left(1+\sqrt{\pi} \frac{\sqrt{\varepsilon^{2} / 3+2 \sigma^{2}}-\varepsilon / \sqrt{3}}{\varepsilon}\right),
\end{aligned}
$$

where $\sigma$ denotes the standard deviation of the Gaussian observational noise. The term $[\kappa+b \ln (1-\alpha \varepsilon)]$ represents the approximate form of $K_{2}(\varepsilon) ; \kappa$ is the correlation entropy; $b \ln (1-\alpha \varepsilon)$ indicates the effect of the coarse graining. Here, we define $g(\cdot)$ as follows:

$$
g(v)=\frac{2}{\sqrt{\pi}} \frac{v \exp \left(-v^{2}\right)}{\operatorname{Erf}(v)}
$$

where $\operatorname{Erf}(v)$ shows the error function defined as

$$
\operatorname{Erf}(v)=\frac{2}{\sqrt{\pi}} \int_{0}^{v} \exp \left(-t^{2}\right) \mathrm{d} t
$$

We fit parameters $\kappa, \alpha, b$, and $\sigma$ by using Eq. (15) based on given time series data, so that a bimodal distribution appears on an $\left(\varepsilon, K_{\text {noisy }}(\varepsilon) \varepsilon^{\mu}\right)$-plane if the time series is influenced by the Gaussian observational or dynamical noise, where $\mu$ is a constant of order 1 . Consequently, the first local maximum value corresponding to the smaller $\varepsilon$ is equivalent to $\sigma$ if

$$
\mu \approx 3.441717-\frac{1}{\ln \sigma},
$$

which has been numerically found by Urbanowicz and Holyst [9].

\subsection{Examples}

Here, we discuss some examples of short time series for which Urbanowicz and Holyst's method does not produce estimates that sufficiently correlate with the actual dynamical noise levels and universally work among systems [see Figs. 2(a)-(d)]. In these examples, the relationship between the estimated and the actual levels of dynamical noise has been investigated numerically with the use of their method [9]. Time series data (sampled observational signals) were generated with four types of systems, each of which was influenced by dynamical 


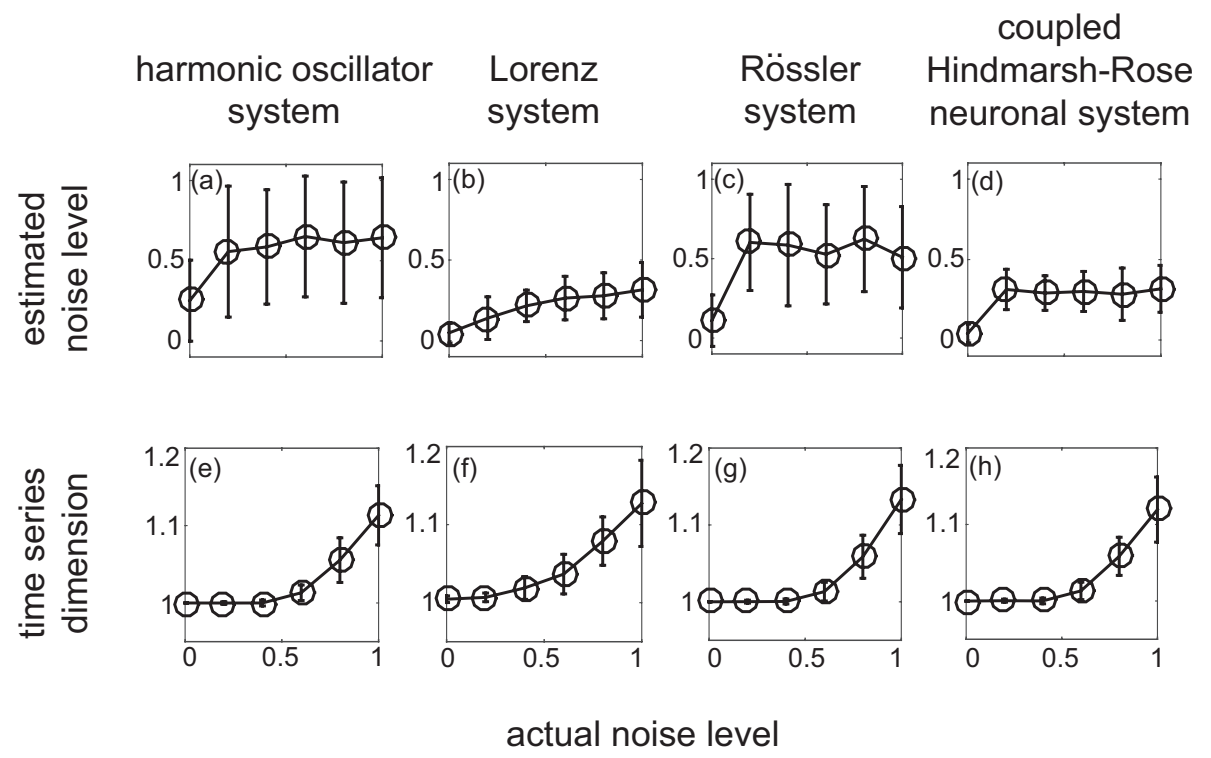

Figure 2: (a) to (d): Relationship between the noise level estimated with Urbanowicz and Holyst's method and the actual dynamical noise level. (e) to (h): Relationship between the time series dimension (TSD) and the actual dynamical noise level. Time series data are generated for (a, e) the harmonic oscillatory system [Eqs. (18)-(20)], (b, f) the Lorenz system [Eqs. (21)-(24)], (c, g) the Rossler system [Eqs. (25)-(28)], and (d, h) the coupled Hindmarsh-Rose neuronal system [Eqs. (29)-(32)]. noise $\rho_{w} \mathrm{~d} W_{w}(t)\left(w \in\left\{\mathrm{x}, \mathrm{y}, \mathrm{z}, \mathrm{x}_{n}, \mathrm{y}_{n}, \mathrm{z}_{n}\right\}\right)$ and converted to observational signals ${ }_{157}\left\{X_{\mathrm{obs}}(t)\right\}:$

(I) The harmonic oscillator system was simulated with

$$
\begin{aligned}
\mathrm{d} x & =y \mathrm{~d} t+\rho_{\mathrm{x}} \mathrm{d} W_{\mathrm{x}}(t), \\
\mathrm{d} y & =-x \mathrm{~d} t+\rho_{\mathrm{y}} \mathrm{d} W_{\mathrm{y}}(t), \\
X_{\text {obs }}(t) & =x(t)+\sigma_{\mathrm{X}} \xi_{\mathrm{X}}(t) .
\end{aligned}
$$


(II) The Lorenz system was simulated with

$$
\begin{aligned}
\mathrm{d} x & =[-10 x+28 y] \mathrm{d} t+\rho_{\mathrm{x}} \mathrm{d} W_{\mathrm{x}}(t), \\
\mathrm{d} y & =[-x z+28 x-y] \mathrm{d} t+\rho_{\mathrm{y}} \mathrm{d} W_{\mathrm{y}}(t), \\
\mathrm{d} z & =[x y-(8 / 3) z] \mathrm{d} t+\rho_{\mathrm{z}} \mathrm{d} W_{\mathrm{z}}(t), \\
X_{\mathrm{obs}}(t) & =x(t)+\sigma_{\mathrm{X}} \xi_{\mathrm{X}}(t) .
\end{aligned}
$$

(III) The Rössler system was simulated with

$$
\begin{aligned}
\mathrm{d} x & =[-y-z] \mathrm{d} t+\rho_{\mathrm{x}} \mathrm{d} W_{\mathrm{x}}(t), \\
\mathrm{d} y & =[x+0.36 y] \mathrm{d} t+\rho_{\mathrm{y}} \mathrm{d} W_{\mathrm{y}}(t), \\
\mathrm{d} z & =[0.4+z(x-4.5)] \mathrm{d} t+\rho_{\mathrm{z}} \mathrm{d} W_{\mathrm{z}}(t), \\
X_{\mathrm{obs}}(t) & =x(t)+\sigma_{\mathrm{X}} \xi_{\mathrm{X}}(t) .
\end{aligned}
$$

(IV) The coupled Hindmarsh-Rose neuronal system [12] with 100 neurons, the $n$th neuron of which was influenced by the external input $I_{n}$, was simulated with

$$
\begin{aligned}
\mathrm{d} x_{n} & =\left[y_{n}-x_{n}^{3}+3 x_{n}^{2}-z_{n}+I_{n}+\sum_{\gamma \neq n}^{100} 0.05 \theta\left(x_{\gamma}\right)\right] \mathrm{d} t+\rho_{\mathrm{x}_{n}} \mathrm{~d} W_{\mathbf{x}_{n}}(t), \\
\mathrm{d} y_{n} & =\left[1-5 x_{n}^{2}-y_{n}\right] \mathrm{d} t+\rho_{\mathrm{y}_{n}} \mathrm{~d} W_{\mathrm{y}_{n}}(t), \\
\mathrm{d} z_{n} & =\left[0.006\left\{4\left(x_{n}+1.6\right)-z_{n}\right\}\right] \mathrm{d} t+\rho_{\mathrm{z}_{n}} \mathrm{~d} W_{\mathrm{z}_{n}}(t), \\
X_{\mathrm{obs}}(t) & =x_{1}(t)+\sigma_{\mathrm{X}} \xi_{\mathrm{X}}(t),
\end{aligned}
$$

see Eq. (13) for $\theta(\cdot)$, where the amount $\rho_{w}$ of the dynamical noise was constant over time. The stochastic variable $\mathrm{d} W_{w}$ follows a normal distribution of mean 0 and standard deviation $\sqrt{\mathrm{d} t}$. For systems (I) through (IV), the observational signal $\left\{X_{\mathrm{obs}}(t)\right\}$ has been used to test Urbanowicz and Holyst's method, and for simplicity, we have assumed that the signal is independent of observational noise $\sigma_{\mathrm{X}} \xi_{\mathrm{X}}(t)$ by setting $\sigma_{\mathrm{X}}=0$. Actually, in the network composed of the Hindmarsh-Rose neurons [Eqs. (29) to (32)], a synchronized chaotic oscillation appears [13]. Each value of $\left\{I_{n}\right\}$ has been uniformly distributed between 1 and 
5. Fifty time series of $\left\{X_{\text {obs }}(t)\right\}$ were simulated with respect to each noise level, which is defined as $\rho_{w} / \sigma_{\mathrm{dyn} w}$. Here $\sigma_{\mathrm{dyn} w}$ indicates the standard deviation of the variable $w$, and the initial condition of $w$ follows the uniform distribution between 0 and 1 for each noise level. Note that $\sigma_{\mathrm{dyn} w}$ is not time-dependent because the simulation was conducted repeatedly over multiple trials. The sampling time $\Delta t$ was set to 0.001 , and the length $L_{\text {seg }}(0, \Delta t)$ of each time series was set to 100 points, in which the transient state following the onset of the simulation was included in the tested time series, i.e., $t_{0}=0$ hereafter.

The time series with length of $L_{\mathrm{seg}}(0, \Delta t)=100$ were identified as short time series by calculations of the quantities $I_{1}(c)$ and $I_{2}(c)$ [Eq. (4) and Eq. (5)], in which $\mathrm{E}_{\tau}[\cdot]$ and $\operatorname{Var}_{\tau}[\cdot]$ were calculated with an analysis window $h^{\prime}=0.005$. Then, $h$ was set to 0.001 so that a partially observational signal $\left\{X_{\text {obs }}(s)\right\}$ over $\tau \leq s \leq \tau+h+h^{\prime}$ can be considered as a semi-stationary process. The upper bounds $\epsilon_{1}$ and $\epsilon_{2}$ [see Eq. (8) and Eq. (9)] have been chosen as follows: $\epsilon_{1}=$ 1 and $\epsilon_{2}=0.05$. For these values, the corresponding threshold values of $c^{*}$ [Eq. (10)] for the four systems given in Eqs. (18) to (32) were 1878.07 $\Delta t$, 103.41 $\Delta t, 229.14 \Delta t$, and $122.33 \Delta t$, respectively. In the calculation of $c^{*}$ for each system, we have used 100 time series of $\left\{X_{\mathrm{obs}}(t)\right\}$ with $L_{\mathrm{seg}}(0, \Delta t)=50000$. Consequently, for each system we have obtained 100 threshold values for $c^{*}$. Finally, the average of these values has been considered as the final threshold value $c^{*}$. When we estimated $c^{*}$, each system was affected by dynamical noise with the noise level of 0.1 and simulated for different initial conditions (uniformly distributed between 0 and 1) over 100 trials.

A system with any noise level $\rho_{w} / \sigma_{\operatorname{dyn} w}$ was simulated in two steps: first, a clean system, without any noise, was simulated with an initial condition $\vec{x}_{0}$, and the resulting standard deviation $\sigma_{\mathrm{dyn} w}$ was stored in memory; next, the system including dynamical noise was simulated using the same initial condition $\vec{x}_{0}$ with the clean system, and then the noise level was set to the same values over $x, y$, and $z$ or over $\left\{x_{n}\right\},\left\{y_{n}\right\}$, and $\left\{z_{n}\right\}$, where $\rho_{w}$ was obtained by multiplication between the arbitrarily set noise level and the stored $\sigma_{\text {dyn } w}$.

In Urbanowicz and Holyst's method, we have defined the estimated noise 
level as $\hat{\sigma} / \sigma_{\text {dyn } w}$, where $\hat{\sigma}$ represents the fitted standard deviation of the Gaussian observational noise [see Eq. (15)]. Clearly, the numerical results presented in Fig. 2 reveal that Urbanowicz and Holyst's method is not necessarily suitable for estimating the level of dynamical noise and for functioning universally among systems.

\section{Proposed method for noise-level estimation}

In this section, a novel method for detecting changes in the level of dynamical noise is presented. Here consider two types of time series: (1) A series originating from a flow generated from a continuous-time dynamical system; (2) A series originating from a map generated from a discrete-time dynamical system. The proposed method can be applied to the former time series in the real world by calculating the fractal dimension developed by Higuchi [14], even if the dynamical system is unknown. To avoid confusing the fractal dimension with the correlation dimension in the reconstructed attractor, we call the fractal dimension using the Higuchi method as the time series dimension (TSD) because the Higuchi method considers a time series to be a figure represented on a two-dimensional space, in which the horizontal and vertical axes denote time and amplitude, respectively.

Here we assume that an observational signal is sampled with the time interval $\Delta t>0$. Then, the TSD of the resulting time series reflects both the deterministic and stochastic components of the time series, and ranges from 1 to 1.5. When the dynamical system underlying the time series contains only the deterministic component, the TSD of the time series is 1 because the series locally resembles a line. In contrast, when the dynamical system underlying the time series contains only the stochastic component that represents the Gaussian white noise, the TSD of the time series is 1.5 , the fractal dimension of the Wiener process [15]. Therefore, the relative dominance ratio between the deterministic and stochastic components determines the TSD.

We stress the fact that in the present study, our focus is on a system [see 
Eq. (1)], in which the deterministic and stochastic components are mixed. Furthermore, since the observed signal [Eq. (2)] is sampled with the certain time interval, the corresponding TSD depends not only on the amount of dynamical noise but also on the sampling time $\Delta t$. If the deterministic component does not contribute to the system [Eq. (1)] at all, the TSD is 1.5, independent of the amount of dynamical noise and of the sampling time, as mentioned above. Further, the TSD does not require the power law.

In Subsection 4.1, we explain how to calculate the curve length of a given time series from a stochastic dynamical system of the form [Eq. (1)] by introducing the Higuchi method [14]. In Subsection 4.2, we convert the curve length directly to the TSD. The target time series is the sampled signal $\left\{X_{\vec{\rho}}(i \Delta t)\right\}$ $(i=1,2, \ldots, N)$ of the variable $x_{q}$ perturbed by dynamical noise with $\vec{\rho}(t)=$ $\left(\rho_{1}(t), \rho_{2}(t), \ldots, \rho_{d}(t)\right)$, or more precisely, with $\vec{\rho}(i \Delta t)$; namely, it has been assumed that $\left\{X_{\vec{\rho}}(i \Delta t)\right\}$ is influenced by dynamical noise, not by observational noise. Hereinafter, we denote $\vec{\rho}(i \Delta t)$ as $\vec{\rho}$, for simplicity. Finally, in Subsection 4.3 , we present a numerical investigation of the proposed method, in which the level of dynamical noise has been estimated.

\subsection{Curve length of time series}

The Higuchi method [14] is based on the following expression [16] for calculating the curve length,

$$
L^{(q)}(\Delta t, \vec{\rho})=\frac{\sum_{i=1}^{N-1}\left|X_{\vec{\rho}}((i+1) \Delta t)-X_{\vec{\rho}}(i \Delta t)\right|}{\Delta t},
$$

where $L^{(q)}(\Delta t, \vec{\rho})$ is an ideal definition of the curve length, but this definition does not work well when we try to get an appropriate TSD. The Higuchi method solves this problem by course-graining the time series and creating some new time series sampled with $\zeta_{k}=k \Delta t$, in which $k$ is a natural number. A larger $k$ results in a larger sampling time $\zeta_{k}$.

Consequently, in the Higuchi method, the modified curve length $\tilde{L}^{(q)}$ associated with the time series $\left\{X_{\vec{\rho}}(i \Delta t)\right\}$ corresponding to the variable $x_{q}$ is 
where

$$
\begin{aligned}
L_{m}^{(q)}\left(\zeta_{k}, \vec{\rho}\right) & =\left\{\left(\sum_{j=1}^{\left\lfloor\frac{N-m}{k}\right\rfloor}\left|X_{\vec{\rho}}((m+j k) \Delta t)-X_{\vec{\rho}}((m+(j-1) k) \Delta t)\right|\right)\right. \\
& \left.\times \frac{N-1}{\left\lfloor\frac{N-m}{k}\right\rfloor k}\right\} / \zeta_{k} .
\end{aligned}
$$

$$
\tilde{L}^{(q)}\left(\zeta_{k}, \vec{\rho}\right)=\frac{1}{k} \sum_{m=1}^{k} L_{m}^{(q)}\left(\zeta_{k}, \vec{\rho}\right),
$$

Here, the notation $\lfloor\cdot\rfloor$ denotes the rounding down; the term $(N-1) /(\lfloor(N-$ $m) / k\rfloor k)$ normalizes the curve length of a segment; and the variable $L_{m}^{(q)}\left(\zeta_{k}, \vec{\rho}\right)$ represents the curve length of the segment with a starting point $m$. Namely, the process of Eq. (35) constructs $k$ new segments $\left\{X_{k}^{m}(\vec{\rho})\right\}$ with $m=1,2, \ldots, k$, each segment of which is defined by

$X_{k}^{m}(\vec{\rho})=\left\{X_{\vec{\rho}}(m \Delta t), X_{\vec{\rho}}((m+k) \Delta t), X_{\vec{\rho}}((m+2 k) \Delta t), \ldots, X_{\vec{\rho}}\left(\left(m+\left\lfloor\frac{N-m}{k}\right\rfloor k\right) \Delta t\right)\right\}$.

Note that the Higuchi method targets the time series sampled with $\zeta_{k}$, so that the process of Eq. (35) performs division by $\zeta_{k}$ instead of $\Delta t$ [see Eq. (33)]. Then, any curve length of the time series sampled with $\zeta_{k}$ can be calculated by coarse-graining of the time series.

\subsection{Relationship between time series dimension and noise level}

The definition of the TSD is based on the box-counting dimension [15], and is given as follows:

$$
D=\lim _{\phi \rightarrow 0} \frac{\log N(\phi)}{\log (1 / \phi)}
$$

where the variable $N(\phi)$ represents a minimal value of the total number of hyperspheres or the hypercubes with size $\phi$ needed to entirely cover a structure like a coast line. When the size $\phi$ and the variable $N(\phi)$ are substituted with the sampling time $\zeta_{k}$ and the curve length $\tilde{L}^{(q)}\left(\zeta_{k}, \vec{\rho}\right)$ of the time series associated 


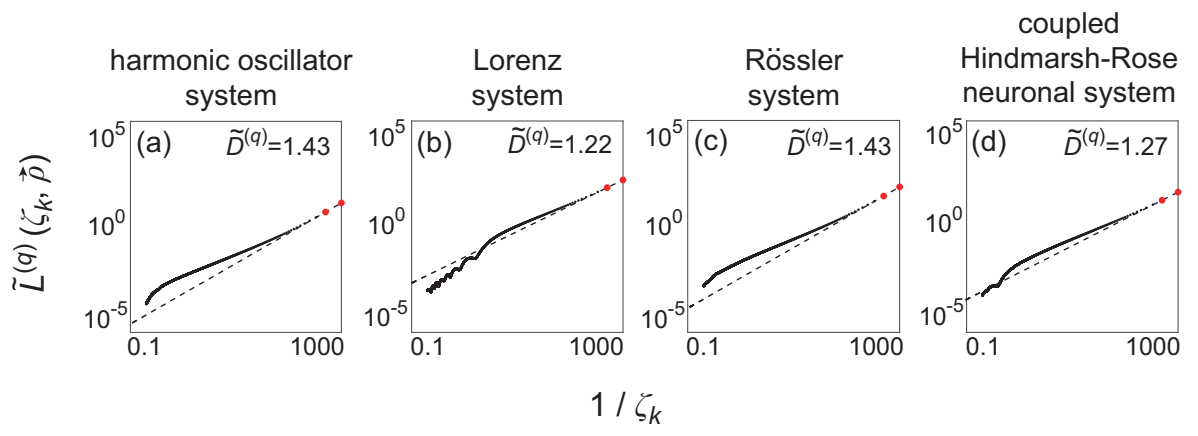

Figure 3: Diverse scaling property of time series data with $L_{\mathrm{seg}}(0,0.001)=10000$, observed through (a) the harmonic oscillatory system, (b) the Lorenz system, (c) the Rössler system, and (d) the coupled Hindmarsh-Rose neuronal system, each system of which was affected by dynamical noise with the noise level of 0.1. For each panel, both the horizontal and vertical axes are displayed by log scales, and the time series dimension (TSD) is calculated as the slope of the approximate line given by only two points $\left(1 / \zeta_{1}, \tilde{L}^{(q)}\left(\zeta_{1}, \vec{\rho}\right)\right)$ and $\left(1 / \zeta_{2}, \tilde{L}^{(q)}\left(\zeta_{2}, \vec{\rho}\right)\right)$, indicated by filled red circles.

with the variable $x_{q}$, respectively, we get an idealized version of the TSD instead of Eq. (37), as follows:

$$
D^{(q)}=\lim _{\Delta t \rightarrow 0} \frac{\log \tilde{L}^{(q)}\left(\zeta_{k}, \vec{\rho}\right)}{\log \left(1 / \zeta_{k}\right)}
$$

We show below the relationship between $D^{(q)}$ and $\vec{\rho}$ based on Eq. (38), where the TSD is converted to an approximate version; note that only the approximate TSD depends on $\vec{\rho}$. For simplicity, we investigate the situation in which only one variable $x_{q}$ of the dynamical system [Eq. (1)] is observed in the observational process and furthermore, we assume that observational noise does not influence the process. The TSD is calculated in the case of $\Delta t \rightarrow 0$ as shown in Eq. (38), so that the TSD is approximated as follows:

$$
D^{(q)} \approx \frac{\log \tilde{L}^{(q)}\left(\zeta_{1}, \vec{\rho}\right)-\log \tilde{L}^{(q)}\left(\zeta_{2}, \vec{\rho}\right)}{\log 2} \equiv \tilde{D}^{(q)}(\vec{\rho}, \Delta t),
$$

where this approximate version is the proposed TSD [see examples in Fig. 3] and becomes a function of $\vec{\rho}$ and of $\Delta t$; hereinafter, we denote this TSD as $\tilde{D}^{(q)}(\vec{\rho})$ because $\Delta t$ is constant throughout this paper, i.e. $\Delta t=0.001$ except for Fig. 9 . 
Note that if $\Delta t$ is small enough so that the time series $\left\{X_{\vec{\rho}}(i \Delta t)\right\}$ behaves like a flow, the difference $\log \tilde{L}^{(q)}\left(\zeta_{1}, \vec{\rho}\right)-\log \tilde{L}^{(q)}\left(\zeta_{2}, \vec{\rho}\right)$ reflects almost entirely the stochastic component, thus the TSD does not depend on $\vec{\rho}$ and always indicates 1.5 , except at $\vec{\rho}=\overrightarrow{0}$. However, in this study we assume that the observational signal is sampled with a certain sampling time $\Delta t$, and consequently, the TSD reflects properties of both the deterministic and stochastic components and ranges between 1 and 1.5 in a certain range of $\vec{\rho}$ [see Subsection 4.3]. Furthermore, it can be seen that despite the fact that the approximate calculation [Eq. (39)] uses only two points to form the power law between $\tilde{L}^{(q)}\left(\zeta_{k}, \vec{\rho}\right)$ and $\zeta_{k}$ [see Fig. 3], these two points might be essential to construct the power law; this view seems to be equivalent to the operation of $\Delta t \rightarrow 0$ included in the ideal definition [Eq. (39)] of the TSD. Namely, the power law tends to converge to a slope formed by the two points as $\Delta t \rightarrow 0$ [see Fig. 3]. Hence, the proposed TSD becomes a more flexible version of the conventional Higuchi's fractal dimension (which handles only fractal signals) and can be applied to any system.

Using Eqs. (34), (35), and (39), we can calculate the TSD. If the righthand side of Eq. (39) is smooth with respect to $\rho_{r}(r=1,2, \ldots, d)$, we can compute its derivative in order to reveal the monotonic relationship between the TSD and the noise level. However, when the Higuchi method is applied to the time series to calculate its curve length, the resulting TSD becomes a non-smooth function because the absolute function is contained in the curve length calculation [Eq. (35)] and consequently, the derivative of Eq. (39) does not exist at certain points. In order to circumvent this problem, we conduct piecewise differentiation, excluding the non-smooth set defined as follows:

$$
\begin{aligned}
\Omega_{r}^{*}= & \left\{\rho_{r} \mid \lim _{\Delta \rho_{r} \rightarrow+0} \frac{\tilde{D}^{(q)}\left(\vec{\rho}+\Delta \vec{\rho}_{r}\right)-\tilde{D}^{(q)}(\vec{\rho})}{\Delta \rho_{r}} \neq \lim _{\Delta \rho_{r} \rightarrow-0} \frac{\tilde{D}^{(q)}\left(\vec{\rho}+\Delta \vec{\rho}_{r}\right)-\tilde{D}^{(q)}(\vec{\rho})}{\Delta \rho_{r}}\right\} \\
& \cup\{-\infty, \infty\},
\end{aligned}
$$

where $\Delta \vec{\rho}_{r}$ is a small deviation around $\rho_{r}$ such that $\Delta \vec{\rho}_{r}=\left(0,0, \ldots, \Delta \rho_{r}, \ldots, 0,0\right)$ 
and implicitly depends on time. Using the following set

$$
\begin{array}{r}
\Omega_{r}^{(S, E)}=\left\{\rho_{r} \mid \quad \lim _{\Delta \rho_{r} \rightarrow+0} \frac{\tilde{D}^{(q)}\left(\vec{\rho}+\Delta \vec{\rho}_{r}\right)-\tilde{D}^{(q)}(\vec{\rho})}{\Delta \rho_{r}}=\lim _{\Delta \rho_{r} \rightarrow-0} \frac{\tilde{D}^{(q)}\left(\vec{\rho}+\Delta \vec{\rho}_{r}\right)-\tilde{D}^{(q)}(\vec{\rho})}{\Delta \rho_{r}},\right. \\
\left.S<\rho_{r}<E, S<E, S \in \Omega_{r}^{*}, E \in \Omega_{r}^{*},(S, E) \cap \Omega_{r}^{*}=\emptyset\right\},
\end{array}
$$

which sorts the differentiable domains, we can perform piecewise differentiation of Eq. (39) by $\rho_{r} \in \Omega_{r}^{(S, E)}$ as follows:

$$
\frac{\partial \tilde{D}_{\mathrm{pw}}^{(q)}(\vec{\rho})}{\partial \rho_{r}}=\frac{1}{\log 2}\left[\frac{1}{\tilde{L}_{\mathrm{pw}}^{(q)}\left(\zeta_{1}, \vec{\rho}\right)} \frac{\partial \tilde{L}_{\mathrm{pw}}^{(q)}\left(\zeta_{1}, \vec{\rho}\right)}{\partial \rho_{r}}-\frac{1}{\tilde{L}_{\mathrm{pw}}^{(q)}\left(\zeta_{2}, \vec{\rho}\right)} \frac{\partial \tilde{L}_{\mathrm{pw}}^{(q)}\left(\zeta_{2}, \vec{\rho}\right)}{\partial \rho_{r}}\right] .
$$

Subindex "pw" indicates that the functions $\tilde{D}^{(q)}(\vec{\rho})$ and $\tilde{L}^{(q)}\left(\zeta_{k}, \vec{\rho}\right)$ are piecewisely and sufficiently smooth over interval $(S, E)$ for $\rho_{r}$. If $r \neq q$ [see Eq. $(1)]$, the TSD $\tilde{D}^{(q)}(\vec{\rho})$ does not change with $\rho_{r}$ because the dynamical noises $\left\{\rho_{r}(t) \mathrm{d} W_{r}(t)\right\}_{r \neq q}$ are integrated twice over $t$ when they influence the variable $x_{q}$ and the integrated Wiener process becomes smooth; namely, the derivative of $\tilde{D}_{\mathrm{pw}}^{(q)}(\vec{\rho})$ with respect to $\rho_{r}$ is zero. Consequently, if the following inequality

$$
\frac{\partial \tilde{D}_{\mathrm{pw}}^{(q)}(\vec{\rho})}{\partial \rho_{q}}>0
$$

is satisfied over the concatenation of $S$ and $E$, and if $\tilde{D}^{(q)}(\vec{\rho})$ is continuous with respect to $\rho_{q}$, then $\tilde{D}^{(q)}(\vec{\rho})$ increases with $\rho_{q}$, so that the noise level can be estimated by the TSD. Here, we can easily prove that the absolute function within $\tilde{L}^{(q)}\left(\zeta_{k}, \vec{\rho}\right)$, see Eq. (35), is continuous with respect to $\rho_{q}$ by using the $(\epsilon, \delta)$-definition of limit [17] and consequently, $\tilde{L}^{(q)}\left(\zeta_{k}, \vec{\rho}\right)$ is a continuous function. Note that the inner operation, the increment of $X_{\vec{\rho}}(i \Delta t)$, of the absolute function is also continuous [Eq. (35)] because $X_{\vec{\rho}}(i \Delta t)$ continuously changes with the dynamical noise level.

Further, we analytically derive an inequality including $\Delta t, \vec{\rho}$, and the length $L_{\text {seg }}(0, \Delta t)$ of the time series for the case of linear stochastic dynamical systems [see Appendix B]. Moreover, we summarize our analytic result in the form of a proposition related to the monotonic relationship between the TSD and 


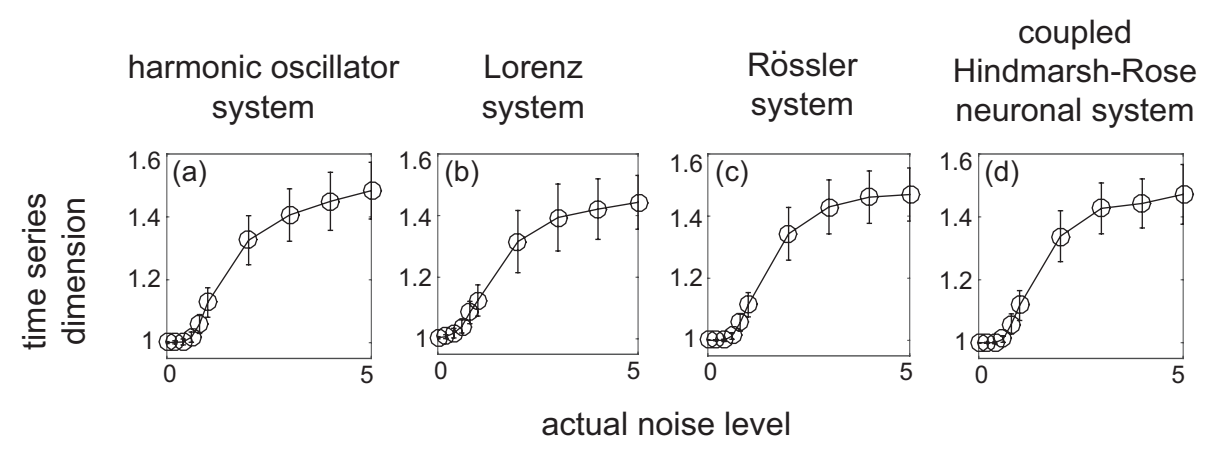

Figure 4: Relationship between the time series dimension (TSD) and the actual dynamical noise level, which ranges from 0 to 5 . Time series data are generated by (a) the harmonic oscillator system, (b) the Lorenz system, (c) the Rössler system, and (d) the coupled Hindmarsh-Rose neuronal system, respectively, over 100 trials for each noise level.

the noise level [see Proposition B.1 in Appendix B]. Additionally, the monotonic relationship between $\tilde{D}^{(q)}(\vec{\rho})$ and $\rho_{q}$ on the $\left(L_{\mathrm{seg}}(0, \Delta t), \rho_{q} / \sigma_{\mathrm{dyn} x_{q}}\right)$-plane is numerically illustrated for the case of a harmonic oscillator.

\subsection{Numerical investigation}

In the proposed method, we can detect changes in the level of dynamical noise by using the TSD. Here, we numerically investigate the relationship between the TSD and the noise level in (I) the harmonic oscillator system [Eqs. (18) to (20)], (II) the Lorenz system [Eqs. (21) to (24)], (III) the Rössler system [Eqs. (25) to (28)], and (IV) the coupled Hindmarsh-Rose neuronal system [Eqs. (29) to (32)], through which we observed short time series of $\left\{X_{\mathrm{obs}}(t)\right\}$ with the length $L_{\text {seg }}(0, \Delta t)=100$, and generated TSDs among 50 trials to create the monotonic properties, with respect to each noise level. As well as in Subsection 3.2 , the transient state following the onset of the simulation was included in the tested time series and the initial condition of variables for each trial followed uniform distributions between 0 and 1. The obtained results, depicted in Figs. 2(e) through $2(\mathrm{~h})$, show that the TSD increases with the noise level, which ranges from 0 to 5 [see Fig. 4], and further, the detailed monotonically increasing properties are similar among systems, so that there exists a possibility that 


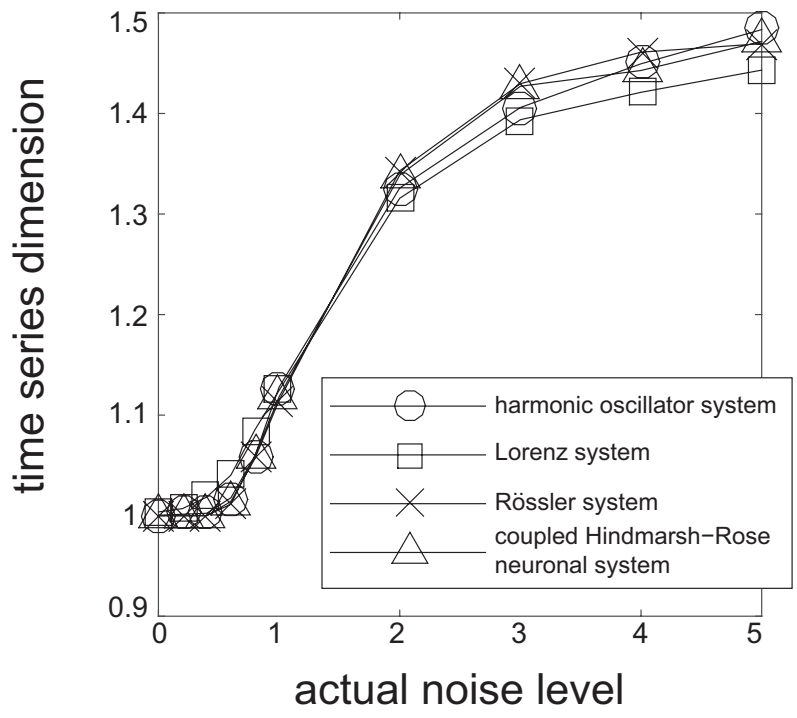

Figure 5: An illustrative comparison among systems' monotonic properties of the time series dimension (TSD) with respect to the actual dynamical noise level of short time series, observed through four types of systems: the harmonic oscillator system, the Lorenz system, the Rössler system, and the coupled Hindmarsh-Rose neuronal system. Time series data used here are the same with Fig. 4.

the TSD can be a universal value related to the dynamical noise level [see Fig. $5]$.

However, if the length of the time series increases to include properties of the respective system, the monotonic behavior differs among systems, as shown in Fig. 6. Actually, we displayed the $\left(L_{\mathrm{seg}}(0, \Delta t), \rho_{w} / \sigma_{\mathrm{dyn} w}\right)$ diagrams to reveal the effect of the length of time series on the monotonic behavior for the four systems. Each parameter point on the $\left(L_{\mathrm{seg}}(0, \Delta t), \rho_{w} / \sigma_{\mathrm{dyn} w}\right)$ diagram indicates the average value of 100 TSDs calculated from time series generated from the corresponding system; namely, for each point in the $\left(L_{\mathrm{seg}}(0, \Delta t), \rho_{w} / \sigma_{\mathrm{dyn} w}\right)$ plane, we generated TSDs among 100 trials as well as Fig. 4, where each simulation has been conducted for initial conditions following the uniform distribution between 0 and 1 . In particular, the diagrams for the Lorenz system and for the coupled Hindmarsh-Rose neuronal system show specific monotonic 


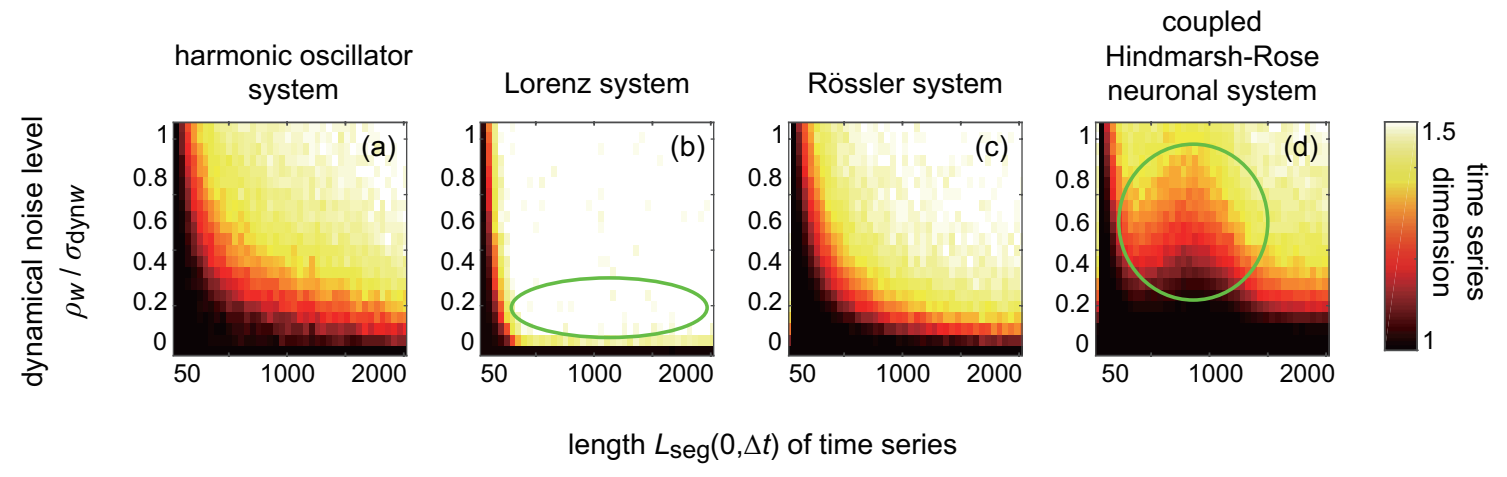

Figure 6: The effect of the length of time series and the dynamical noise level on the monotonic properties among systems: (a) the harmonic oscillatory system, (b) the Lorenz system, (c) the Rössler system, and (d) the coupled Hindmarsh-Rose neuronal system. A larger time series dimension (TSD) is indicated by a lighter color. The effect resulting from the length differs among systems, as shown in areas indicated by open green circles, due to a reflection of systems' own properties to the TSD.

behavior, which is different from the other systems [see Figs. 6(b) and 6(d)]. Note, however, that the TSD can still estimate the noise level for each system. In conclusion, the TSD can estimate the level of dynamical noise for a system on the basis of a monotonic relation.

\section{Application to EEG data}

We applied the proposed method to human EEG data [see Fig. 7]. Two healthy subjects gave written informed consent prior to participation in the experiment. The EEG study was approved by the ethics committees of RIKEN and the University of Tokyo. EEG signals from 63 electrodes were obtained during eyes-open and eyes-closed resting periods. We ran the experiment in a sound-proof, dimly lit room. A cycle of the measurement consists of the eyesopen and eyes-closed periods, each of length $35 \mathrm{~s}$. The cycle of $70 \mathrm{~s}$ was repeated 10 times for two subjects. Sound stimuli ( $500 \mathrm{~Hz}$ or $1 \mathrm{kHz}$ beep) were used to ask subjects to open or close their eyes. Subjects were required to be fixated on a white cross on a black background on a CRT display in the eyes-open condition. 


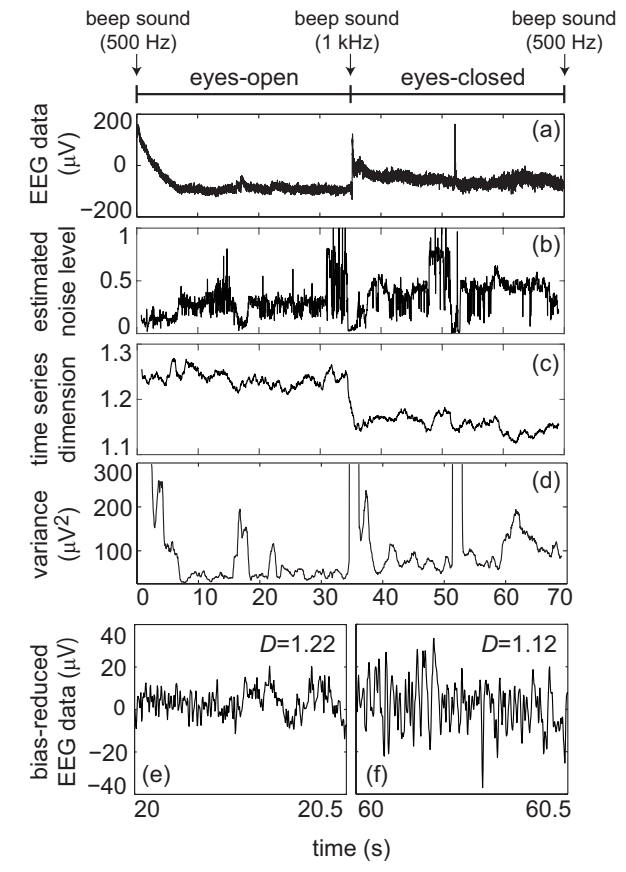

Figure 7: (a) A representative EEG signal obtained from electrode Fp2 during the eyes-open and eyes-closed resting periods. (b) The estimated noise level with Urbanowicz and Holyst's method. (c) The time series dimension (TSD), and (d) the variance. The arrows indicate the onset of the eyes-open or eyes-closed state. (e) A typical time series of the eyes-open state, the TSD of which was 1.22. (f) A typical time series of the eyes-closed state, the TSD of which was 1.12 .

The reference electrodes were placed on both the left and right earlobes, and the sampling frequency was set to $1 \mathrm{kHz}$. Then, the frequencies of the low- and high-pass filters were set to $250 \mathrm{~Hz}$ and $0.016 \mathrm{~Hz}$, respectively.

When we applied Urbanowicz and Holyst's method and our proposed method to the EEG signals, we used an analysis window with 1500 points, shifting along the time axis every 30 points [see Figs. $7(\mathrm{~b})$ and $7(\mathrm{c})$ ]. No preprocessing was performed.

By applying the proposed method, we found that the difference between the eyes-open and eyes-closed brain states becomes evident [see Fig. 7(c)]. Analysis of the 63 time series of the EEG signals showed that the TSD of electrode 
Fp2 can be used to robustly discriminate between the eyes-open and eyes-closed states. With respect to each subject, the Wilcoxon rank sum test was applied to two datasets: TSDs (11400 points) of the eyes-open period and TSDs (11400 points) of the eyes-closed period, both of which include 10 trials; 1140 TSDs were calculated in the eyes-open or eyes-closed periods for each trial. The results showed that the median of the eyes-open dataset was significantly greater than that of the eyes-closed dataset $(P<0.0001$ for two subjects $)$; medians of the eyes-open and eyes-closed datasets were 1.24 and 1.18 for subject 1 and 1.19 and 1.16 for subject 2 , respectively. The TSD is less influenced by disturbances such as eye movement [see Fig. 7(c)] so that the TSD of each state of the eyes-open and eyes-closed is temporally stable, i.e., if we set an appropriate threshold value, each state can be definitely distinguished. In contrast, Urbanowicz and Holyst's method did not clearly discriminate between the eyesopen and eyes-closed states [see Fig. 7(b)]. The variance of the time series also differed between the eyes-open and eyes-closed states [see Fig. 7(d)], where an analysis window similar to those applied to Figs. 7(b) and 7(c), was used for dynamically obtaining the variance, but the difference between the states was smaller than that using the TSD [see Fig. 7(c)]. Moreover, the variance is very sensitive to disturbances so that the time series of the variance fluctuates over a wide range of amplitudes. Thus, the proposed method can estimate the level of the dynamical noise more reliably and distinguish the eyes-open and eyes-closed states robustly.

\section{Discussions}

A novel dimension called TSD, for estimating the level of dynamical noise perturbing in time series, observed through stochastic dynamical systems has been proposed. We have defined the dynamical noise in the sense of the Wiener process, and based on this definition, it has been validated whether a monotonic relationship between the TSD and the dynamical noise level is achieved among four specific systems [see Subsection 4.3]. The TSD may be a well-defined 
dimension because it has been derived directly from the scaling property [see Fig. 3] of the conventional Higuchi's fractal dimension so that the TSD does not require the power law and is applicable to non-fractal signals. Furthermore, the applicability of the TSD to real time-series data has been demonstrated with the application of the proposed method to human EEG signals. The obtained results have shown that the estimated TSD temporally changes due to visual inputs [see Fig. 7(c)].

Possibly due to the noise-induced order [18], the variance of the eyes-opened period was low compared to that of the eyes-closed period [see Fig. $7(\mathrm{~d})$ ]. In particular, the dynamical neural noise caused by the light stimulus may play a role in realizing the noise-induced order. Typical time series [see Figs. 7(e) and $7(\mathrm{f})$ ] of the eyes-open and eyes-closed states have implied that the TSD reflects the relative dominance ratio between the deterministic and stochastic components. In the case of the eyes-open state [see Fig. 7(e)], the TSD was larger corresponding with miniature fluctuations in the EEG data. In the case of the eyes-closed state [see Fig. 7(f)], the TSD was smaller corresponding with large fluctuations in the EEG data.

The proposed TSD may also work as an effective indicator of dynamical noise even for transient time series data (containing transient components). We have supported our claim with the numerical analysis presented in Subsection 4.3. In particular, short time series with the length $L_{\mathrm{seg}}(0, \Delta t)=100$, obtained from four different systems, have shown a similar monotonically increasing property of the TSD with respect to the dynamical noise level, as depicted in Fig. 4. Thus, the present method may be used to analyze time series containing transient behavior.

On the other hand, if one has multivariate time series, especially two time series, one may be able to adjust their monotonic properties so that the universal monotonicity holds for both of them, by changing the length of time series [see Fig. 8] or by resampling time series [see Fig. 9]. Let us consider the situation that we have two monotonic properties and try to fit these properties with each other. As a particular example, we try to make a monotonic property 


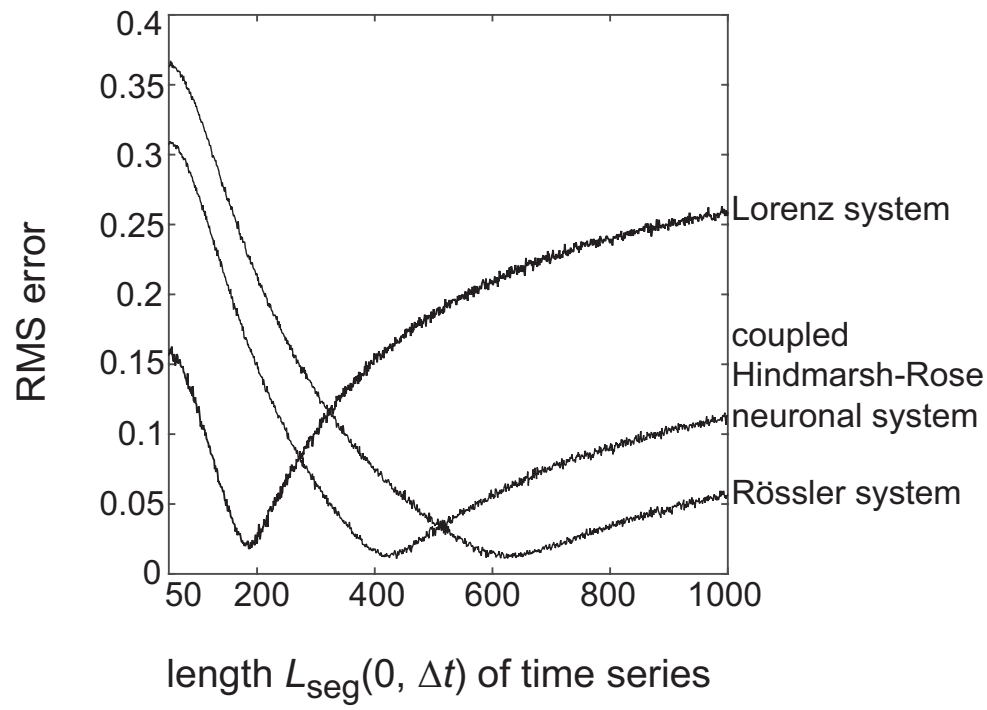

Figure 8: Adjustments of the length of short time series to work the systems universally. A case that the monotonic property of (1) the harmonic oscillator system approaches those of other three systems: (2) the Lorenz system, (3) the Rössler system, and (4) the coupled Hindmarsh-Rose neuronal system. Clearly, the minimal values of the RMS errors correspond to $L_{\mathrm{seg}}(0, \Delta t)=186$ for system $(2), L_{\mathrm{seg}}(0, \Delta t)=626$ for system $(3)$, and $L_{\mathrm{seg}}(0, \Delta t)=437$ for system (4), respectively.

generated from the harmonic oscillator system, equal to each of monotonic properties generated from the Lorenz system, the Rössler system, and the coupled Hindmarsh-Rose neuronal system. The lengths of the time series associated with these four systems have been fixed to 4502, 182, 574, and 407 points, respectively so that the beginning of this simulation has been equivalent to Fig. A1(a) in Appendix A; these lengths correspond to $\left\lfloor c^{*} / \Delta t\right\rfloor$ derived from two upper bounds $\epsilon_{1}=2.5$ and $\epsilon_{2}=0.07$ [see Eqs. (8) and (9)], where $\lfloor\cdot\rfloor$ denotes the rounding down. As the length $L_{\mathrm{seg}}(0, \Delta t)=4502$ of the time series decreases, the root mean square (RMS) errors [see Eq. (A1)] between two systems also decrease, as shown in Fig. 8. Among three pairs of two systems (i.e. (i) the harmonic oscillator system vs. the Lorenz system, (ii) the harmonic oscillator system vs. the Rössler system, and (iii) the harmonic oscillator system vs. the 


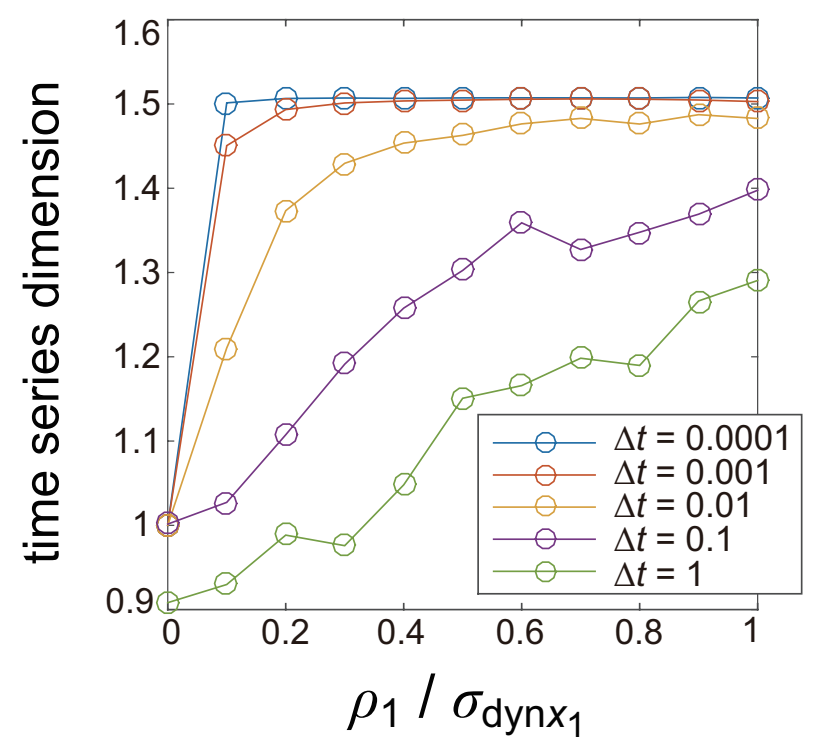

Figure 9: The effect of the sampling time $\Delta t$ on the monotonic property corresponding to the harmonic oscillator system. As $\Delta t$ decreases, the time series dimension (TSD) becomes constant along with the noise level larger than 0 . As $\Delta t$ increases, the monotonic property changes, but the property still holds even if $\Delta t=1$.

coupled Hindmarsh-Rose neuronal system), when we change the length of time series associated with the harmonic oscillator system from 4502 to 50, the RMS errors indicate very small values (less than 0.05 ) at certain length of time series, see Fig. 8. The lengths corresponding to the minimal values of the RMS errors are 186, 626, and 437 for pairs of systems (i) through (iii), respectively. Thus, we can flexibly control the monotonic properties to work them universally by changing the length of time series.

Finally, we discuss the case of adjusting the monotonic property of a system by resampling the corresponding time series data. We have simulated 100 short time series of $\left\{X_{\mathrm{obs}}(t)\right\}$ with the lengths of $L_{\mathrm{seg}}(0,0.0001 k)=\lceil 45020 / k\rceil$, as well as Fig. 8 when $k=10$, where $\lceil\cdot\rceil$ denotes the rounding up. These time series correspond to the harmonic oscillator system affected by dynamical noise. Five monotonic properties, each of which corresponds to the sampling time 0.0001, 
$0.001,0.01,0.1$, and 1 of $\Delta t$, respectively, have been generated. At this point it is worth to remember that the TSD actually depends on $\Delta t$ as well as $\vec{\rho}$, although until now we have considered a fixed sampling time $\Delta t=0.001$. Now, consider the monotonic property obtained by using a very small sampling time, namely $\Delta t=0.0001$. This property almost does not depend on the noise level except at $\vec{\rho}=\overrightarrow{0}$, and then the resulting TSD always indicates around 1.5 so that the property cannot work as an indicator of the dynamical noise level. Next, suppose that we resample the time series, which was sampled at $\Delta t=0.001$, by new sampling times of $0.01,0.1$, and 1 , respectively. As $\Delta t$ increases, the monotonic property changes; that is, the slope of the monotonically increasing behavior gradually decreases. If $\Delta t$ is a very large value for example $\Delta t=1$, the monotonic behavior tends to be unstable, but the increasing property still holds. Thus, along with the resampled multivariate time series data in a plausible way, the resulting TSDs may work universally.

\section{Conclusions}

A method, based on the TSD, for detecting changes in the level of the dynamical noise in stochastic dynamical systems has been proposed. It has been shown that the TSD has a monotonic relationship with the level of dynamical noise. This novel TSD is an extended or, rather, a flexible version of the conventional Higuchi's fractal dimension because the TSD can handle any stochastic dynamical system, including non-fractal signals. Furthermore, a theory of the TSD has been presented for the case of linear stochastic dynamical systems. This theory should be extended to cover more general systems, namely systems affected by colored dynamical noise and systems influenced not only by dynamical noise but also by observational noise. In order to show the applicability of the TSD to real-world systems, the proposed method has been applied to EEG data, in which the TSD has shown robustness to sudden disturbances. We believe that the proposed method may help us understand the behavior of various complex dynamical systems, including the brain. 


\section{Acknowledgement}

This work was supported by the Aihara Innovative Mathematical Modelling Project and the Japan Society for the Promotion of Science (JSPS) through its "Funding Program for World-Leading Innovative R\&D on Science and Technology (FIRST Program)," which was initiated by the Council for Science and Technology Policy (CSTP). This work was also supported by JSPS KAKENHI Grant Number 20246026.

\section{Appendix}

\section{A. How to determine the threshold value $c^{*}$ in Eq. (10)}

In this appendix, a procedure for determining the threshold value $c^{*}$ for a given observational signal is presented. This threshold value provides a boundary between short and long time series for the TSD analysis. An optimal length or analysis window for the TSD is assessed, based on the threshold value and accordingly, the time series within the analysis window will be converted to the TSD in turn along the time axis. Actually depending on the length of time series, the function of the TSD can be changed from the viewpoint of the monotonic behavior, which can be seen between the TSD and the level of dynamical noise [Fig. 6]. The diagrams, consisting of the two upper bounds $\epsilon_{1}$ and $\epsilon_{2}$ [Eq. (8) and Eq. (9)] with the monotonic properties, are described. On the diagrams, the similarity among typical monotonic properties - for which the upper bounds can be selected reasonably for a given observational signal - is plotted.

Here we regard a monotonic property consisting of TSDs, as a function of the two upper bounds $\epsilon_{1}$ and $\epsilon_{2}$. Furthermore, assume that $\epsilon_{1}$ and $\epsilon_{2}$ vary from 1 to 9.5 and from 0.01 to 0.095 , respectively; note that if these bounds are set to very large values, they cannot work as the plausible boundary because short time series defined in the sense of this study mean that the time series is referred to as a process close to a semi-stationary process; i.e., it only includes small discrepancies between stationarity and nonstationarity [Fig. 1]. We have 


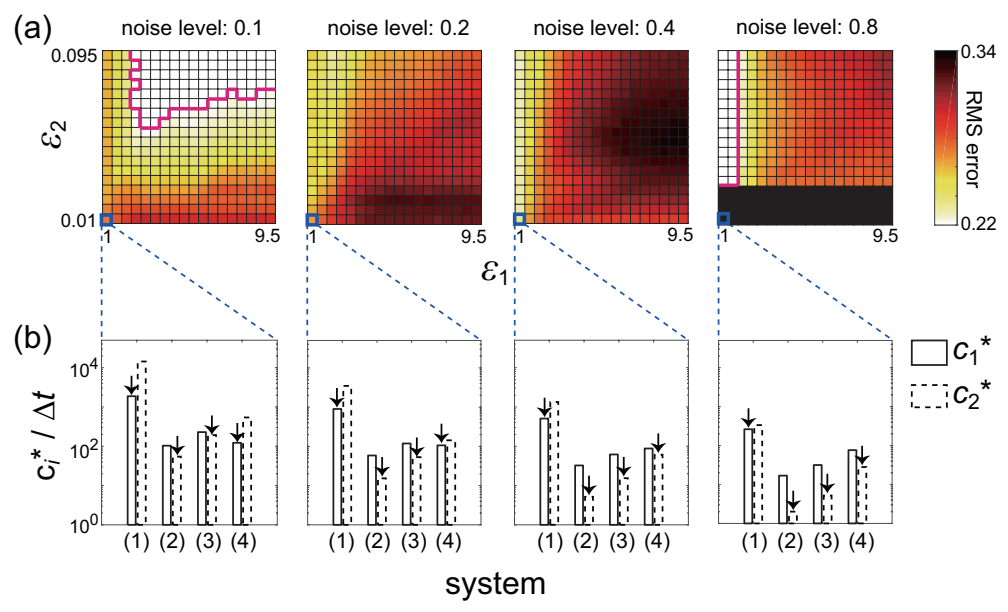

Figure A1: Effects of the two upper bounds $\epsilon_{1}$ and $\epsilon_{2}$, on the monotonic property of the TSD. (a) The $\left(\epsilon_{1}, \epsilon_{2}\right)$ diagrams in accordance with the noise level from left to right: $0.1,0.2,0.4$, and 0.8. The horizontal axis is $\epsilon_{1}$ ranged from 1 to 9.5 in steps of 0.5 , while the vertical axis is $\epsilon_{2}$ ranged from 0.01 to 0.095 in steps of 0.005 . Each parameter point on the diagrams represents the similarity of the monotonic properties among four systems: (1) the harmonic oscillator system, (2) the Lorenz system, (3) the Rössler system, and (4) the coupled Hindmarsh-Rose neuronal system, where the similarity is evaluated by the RMS error so that a smaller error is indicated by a lighter color. The two magenta curves are boundaries of the RMS error of 0.22. (b) Examples of threshold values for $c_{1}^{*}$ and $c_{2}^{*}$ on a point $\left(\epsilon_{1}, \epsilon_{2}\right)=(1,0.01)$, indicated by open blue rectangles in (a). The vertical axes are displayed by the log scale. The arrows indicate the threshold values of $c^{*}$, by which we set the length of short time series and calculated RMS errors for (a); namely, we used $\left\lfloor c^{*} / \Delta t\right\rfloor$ as the length of each short time series.

generated the $\left(\epsilon_{1}, \epsilon_{2}\right)$ diagrams in terms of the similarity among the following four systems: (I) the harmonic oscillator system, (II) the Lorenz system, (III) the Rössler system, and (IV) the coupled Hindmarsh-Rose neuronal system [Eqs. (18) to (32)], depicted in Fig. A1(a), in which each parameter point indicates the similarity among four monotonic properties generated from the systems. 
similarity has been measured by the RMS error defined as follows:

$$
\begin{aligned}
R M S & =\sqrt{\frac{1}{|\Omega|\left(V^{2}-V\right)} \sum_{\omega \in \Omega} \sum_{e=1}^{V} \sum_{f \neq e}\left|\tilde{D}_{\text {mean }}^{(e)}\left(\omega \overrightarrow{\rho_{e}}\right)-\tilde{D}_{\text {mean }}^{(f)}\left(\overrightarrow{\rho_{f}}\right)\right|^{2}}, \\
\tilde{D}_{\text {mean }}^{(e)}\left(\omega \overrightarrow{\rho_{e}}\right) & =\frac{1}{100} \sum_{\lambda=1}^{100} \tilde{D}_{\lambda}^{(e)}\left(\omega \overrightarrow{\rho_{e}}\right),
\end{aligned}
$$

where $\tilde{D}_{\lambda}^{(e)}\left(\omega \overrightarrow{\rho_{e}}\right)$ denotes the TSD of a time series sampled with $\Delta t=0.001$ observed from $d$-dimensional system (e) [Eqs. (18) to (32)], corresponding to the $\lambda$ th simulation; $e$ represents the index of systems (I) through (IV) so that $V=4 ; \omega$ is the level of dynamical noise, which indicates an element of a set $\Omega=\{0,0.1,0.2, \ldots, 1\} ;$ and $\overrightarrow{\rho_{e}}=\left(\sigma_{\text {dyn } x_{1}}^{(e)}, \sigma_{\text {dyn } x_{2}}^{(e)}, \ldots, \sigma_{\text {dyn } x_{d}}^{(e)}\right)$. Then, $\sigma_{\text {dyn } x_{q}}^{(e)}$ for $q=1,2, \ldots, d$ is the standard deviation of a time series associated with the variable $x_{q}$ included in system $(e)$. Here $|\Omega|$ represents the number of elements in $\Omega$, namely $|\Omega|=11$. The size $\left|\overrightarrow{\rho_{e}}\right|$ of $\overrightarrow{\rho_{e}}$ differs among systems; that is, $\left|\overrightarrow{\rho_{1}}\right|$, $\left|\overrightarrow{\rho_{2}}\right|,\left|\overrightarrow{\rho_{3}}\right|$, and $\left|\overrightarrow{\rho_{4}}\right|$ are respectively $2,3,3$, and 300, each of which corresponds to the number of variables contained in system (e) [Eqs. (18) to (32)].

Toward an application of the $\left(\epsilon_{1}, \epsilon_{2}\right)$ diagrams to the real-data analysis, in fact, we have used the lengths of $\left\lfloor c^{*} / \Delta t\right\rfloor$ among the four systems, where $\lfloor\cdot\rfloor$ denotes the rounding down; the length $\left\lfloor c^{*} / \Delta t\right\rfloor$ corresponds to the upper limit value of the length of short time series. For the sake of clarity, the above simulation has reflected transient behavior, where the initial condition of variables follows the uniform distribution between 0 and 1 . We have applied time series data with the following four kinds of noise levels: $0.1,0.2,0.4$, and 0.8 , respectively, to a set of $\epsilon_{1}$ and $\epsilon_{2}$; i.e., we accumulated discrepancies between stationarity and nonstationarity until their accumulation reached $\epsilon_{1}$ or $\epsilon_{2}$ [Eqs. (4) to $(9)]$, so that only the two $\left(\epsilon_{1}, \epsilon_{2}\right)$ diagrams associated with the noise levels of 0.1 and 0.8 show the relatively small RMS errors less than 0.22 [see Fig. A1(a)].

Here we show the two typical points on one $\left(\epsilon_{1}, \epsilon_{2}\right)$ diagram realized with the noise level of 0.1 [Fig. A2(a)] and on the other $\left(\epsilon_{1}, \epsilon_{2}\right)$ diagram realized 

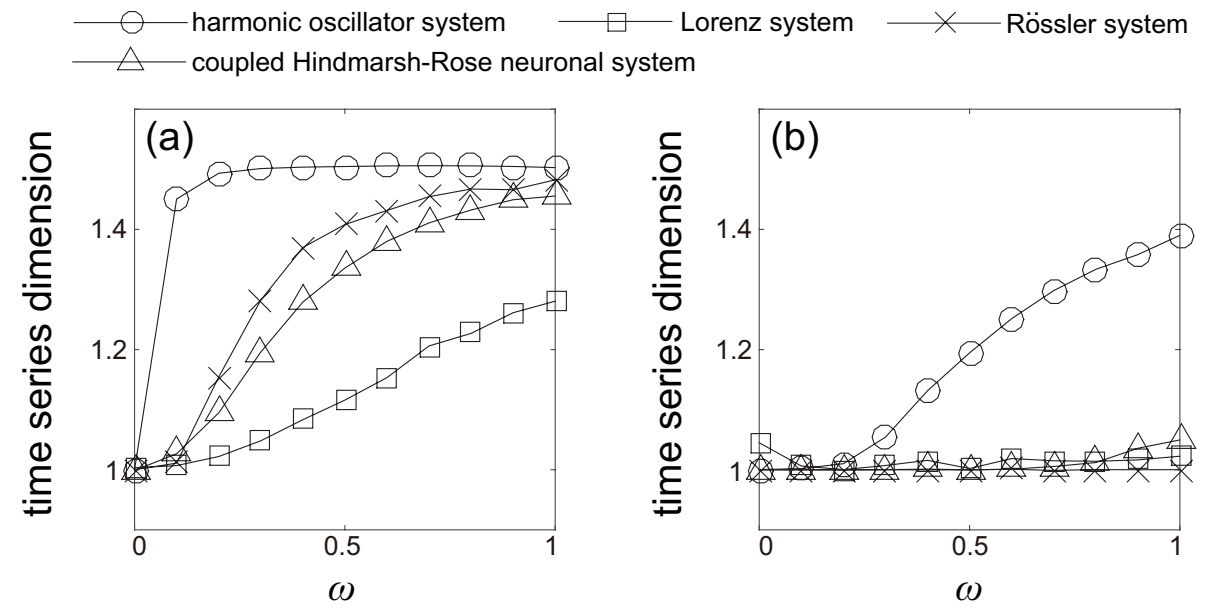

Figure A2: Two examples of monotonic properties among four systems: (1) the harmonic oscillator system, (2) the Lorenz system, (3) the Rössler system, and (4) the coupled HindmarshRose neuronal system, where for both panels (a) and (b), the RMS errors over monotonic properties are less than 0.22 . The lengths of time series used here are obtained as the upper limit values $\left\lfloor c^{*} / \Delta t\right\rfloor$ of short time series, through the uses of $\epsilon_{1}$ and $\epsilon_{2}$ as in Fig. A1. (a) Monotonic behavior. The time series are observed through systems with the noise level of 0.1 , and applied to $\left(\epsilon_{1}, \epsilon_{2}\right)=(2.5,0.07)$. Each length of time series for systems (1) through (4) is $4502,183,574$, and 407, respectively. (b) When the noise level is fixed to 0.8 and $\left(\epsilon_{1}, \epsilon_{2}\right)=(1,0.03)$, monotonic behavior does not appear for all systems. Only the harmonic oscillator has shown the monotonic property. In this case, each length of time series for systems (1) through (4) is $262,4,12$, and 76 , respectively.

with the noise level of 0.8 [Fig. A2(b)]; namely, point $\left(\epsilon_{1}, \epsilon_{2}\right)=(2.5,0.07)$ for noise level 0.1 and point $\left(\epsilon_{1}, \epsilon_{2}\right)=(1,0.03)$ for noise level 0.8. For the noise level of 0.1 , the monotonic behavior can be clearly seen among the four systems [see Fig. A2(a)]. In this case, $\left\lfloor c^{*} / \Delta t\right\rfloor$ of short time series are $4502,183,574$, and 407 for systems (I) through (IV), respectively. On the other hand, for the noise level of 0.8 , the monotonic behavior has not been observed among three systems except for the harmonic oscillator system [see Fig. A2(b)], because in this case, the lengths of short time series are 262, 4, 12, and 76 for systems (I) through (IV), respectively. This irregularity tends to happen when the time 
series applied to $\epsilon_{1}$ and $\epsilon_{2}$ has been affected by the large dynamical noise level so that the length $\left\lfloor c^{*} / \Delta t\right\rfloor$ of short time series, especially generated from the Lorenz system, the Rössler system, and the coupled Hindmarsh-Rose neuronal system, has been very short [see Fig. A1(b)]. Therefore, in order to select the upper bounds $\epsilon_{1}$ and $\epsilon_{2}$-which in turn set the upper limit value of the length of short time series - we should use nearly clean data, i.e. the noise level should be less than 0.1 , especially when we have multivariate time series data and try to work their monotonic properties universally [Fig. 8].

\section{B. Theory of time series dimension}

In this appendix, the monotonic relationship between the TSD $\tilde{D}^{(q)}(\vec{\rho})$ and the standard deviation $\vec{\rho}(t)$ of the time-dependent Gaussian dynamical noise is shown. First, the analytic solution of a linear stochastic dynamical system, obtained via the Laplace transform [19], is introduced. Next, an inequality for the monotonic relationship between the TSD and the noise level, occurring in linear stochastic dynamical systems, is derived. Finally, the success rate of the derived inequality - i.e. the ratio between the number of successful trials satisfying the aforementioned inequality, and the number of total trials - is numerically illustrated in the case of a harmonic oscillator.

\section{B.1. Linear stochastic dynamical system}

The linear stochastic dynamical system with state vector $\vec{x}=\left(x_{1}, x_{2}, \ldots, x_{d}\right)$ is given by

$$
\mathrm{d} x_{q}=\left(\sum_{l=1}^{d} a_{q l} x_{l}\right) \mathrm{d} t+\rho_{q}(t) \mathrm{d} W_{q}(t), \text { for } q=1,2, \ldots, d,
$$

where $\rho_{q}(t)$ is an element of $\vec{\rho}(t)=\left(\rho_{1}(t), \rho_{2}(t), \ldots, \rho_{d}(t)\right)$. This system is the linear version of Eq. (1), where $f_{q}(\vec{x})$ is substituted with the linear combination of $\vec{x} ; a_{q l}$ represents the connection from the variable $x_{l}$ to the variable $x_{q}$. Note that Eq. (B1) denotes a $d$-dimensional linear stochastic dynamical system. 
Under any initial condition, if the temporal change of $\rho_{q}(t)$ is negligible compared to that of the deterministic component, the solution $x_{q}(t)$ of Eq. (B1) is represented by

$$
\begin{aligned}
x_{q}(t) & =F_{q}(t)+\sum_{l=1}^{d} \rho_{l}(t)\left\{G_{q l}(t) * \eta_{l}(t)\right\} \\
& =F_{q}(t)+\sum_{l=1}^{d} \rho_{l}(t) \xi_{q l}(t)
\end{aligned}
$$

where $\eta_{q}(t)=\mathrm{d} W_{q}(t) / \mathrm{d} t$ follows a normal distribution of mean 0 and standard deviation 1. The functions $F_{q}(t)$ and $G_{q l}(t)$ are time-dependent and contribute to the deterministic component and stochastic components, respectively; $G_{q l}(t) *$ $\eta_{l}(t)$ denotes the convolution between the terms $G_{q l}(t)$ and $\eta_{l}(t)$. We cannot give an explicit form of the functions $F_{q}(t)$ and $G_{q l}(t)$, but let us show an example of them by considering the following two-dimensional dynamics:

$$
\begin{aligned}
& \mathrm{d} x_{1}=x_{2} \mathrm{~d} t+\rho_{1} \mathrm{~d} W_{1}(t), \\
& \mathrm{d} x_{2}=-x_{1} \mathrm{~d} t+\rho_{2} \mathrm{~d} W_{2}(t),
\end{aligned}
$$

where we have set $\left(a_{11}, a_{12}, a_{21}, a_{22}\right)=(0,1,-1,0)$ in Eq. (B1). Additionally, we have assumed that $\rho_{1}$ and $\rho_{2}$ are constant over time, for simplicity. By applying the Laplace transform to the above system and considering initial condition $x_{1}(0)=1$ and $x_{2}(0)=0$, we obtain

$$
\begin{aligned}
x_{1}(t) & =\cos (t)+\rho_{1}\left\{\cos (t) * \eta_{1}(t)\right\}+\rho_{2}\left\{\sin (t) * \eta_{2}(t)\right\} \\
& =\cos (t)+\sum_{l=1}^{2} \rho_{l} \xi_{1 l}(t)
\end{aligned}
$$

and

$$
\begin{aligned}
x_{2}(t) & =-\sin (t)-\rho_{1}\left\{\sin (t) * \eta_{1}(t)\right\}+\rho_{2}\left\{\cos (t) * \eta_{2}(t)\right\} \\
& =-\sin (t)+\sum_{l=1}^{2} \rho_{l} \xi_{2 l}(t),
\end{aligned}
$$

for $t \geq 0$. 


$$
\sum_{j=1}^{\left\lfloor\frac{N-m}{k}\right\rfloor}\left|J_{\vec{\rho}}^{(q)}(m, j, k)\right|=\sum_{j \in \Omega_{\mathrm{p} q}(\vec{\rho}, m, k)} J_{\vec{\rho}}^{(q)}(m, j, k)-\sum_{j \in \Omega_{\mathrm{n} q}(\vec{\rho}, m, k)} J_{\vec{\rho}}^{(q)}(m, j, k) .
$$

${ }_{621}$ Next, in order to perform piecewise differentiation of $\tilde{L}^{(q)}\left(\zeta_{k}, \vec{\rho}\right)$ with respect to ${ }_{622} \rho_{q}$, we have removed the non-smooth points by using the set defined as

$$
\Psi^{(q)}(m, k):=\left\{\vec{\rho} \mid \Omega_{\mathrm{zq}}(\vec{\rho}, m, k)=\emptyset\right\},
$$


which sorts the differentiable domains. Consequently, with $\vec{\rho} \in \Psi^{(q)}(m, k)$, Eq. (B9) can be converted to a piecewise smooth function as follows:

$\tilde{L}_{\mathrm{pw}}^{(q)}\left(\zeta_{k}, \vec{\rho}\right)=\frac{(N-1)}{k^{2} \zeta_{k}} \sum_{m=1}^{k}\left[\frac{1}{\left\lfloor\frac{N-m}{k}\right\rfloor}\left\{\sum_{j \in \Omega_{\mathrm{p} q}(\vec{\rho}, m, k)} J_{\vec{\rho}}^{(q)}(m, j, k)-\sum_{j \in \Omega_{\mathrm{n} q}(\vec{\rho}, m, k)} J_{\vec{\rho}}^{(q)}(m, j, k)\right\}\right]$,

where subindex "pw" in the left-hand side has been used to indicate that the function is piecewisely and sufficiently smooth with respect to $\vec{\rho}$. Therefore, its derivative with respect to $\rho_{q}$ exists and is given by

$$
\begin{aligned}
\frac{\partial \tilde{L}_{\mathrm{pw}}^{(q)}\left(\zeta_{k}, \vec{\rho}\right)}{\partial \rho_{q}}= & \frac{(N-1)}{k^{2} \zeta_{k}} \sum_{m=1}^{k}\left[\frac { 1 } { \lfloor \frac { N - m } { k } \rfloor } \left\{\sum_{j \in \Omega_{\mathrm{p} q}(\vec{\rho}, m, k)}\left\{\xi_{q q}((m+j k) \Delta t)-\xi_{q q}((m+(j-1) k) \Delta t)\right\}\right.\right. \\
& \left.\left.-\sum_{j \in \Omega_{\mathrm{n} q}(\vec{\rho}, m, k)}\left\{\xi_{q q}((m+j k) \Delta t)-\xi_{q q}((m+(j-1) k) \Delta t)\right\}\right\}\right],
\end{aligned}
$$

where we have used the fact that elements and sizes of sets $\Omega_{\mathrm{p} q}(\vec{\rho}, m, k)$ and $\Omega_{\mathrm{n} q}(\vec{\rho}, m, k)$ are not changed due to differentiation with respect to $\rho_{q}$; namely, $\Omega_{\mathrm{p} q}(\vec{\rho}, m, k)=\Omega_{\mathrm{p} q}\left(\vec{\rho}+\mathrm{d} \vec{\rho}_{q}, m, k\right)$ and $\Omega_{\mathrm{n} q}(\vec{\rho}, m, k)=\Omega_{\mathrm{n} q}\left(\vec{\rho}+\mathrm{d} \vec{\rho}_{q}, m, k\right)$ where $\mathrm{d} \vec{\rho}_{q}$ is a small deviation around $\rho_{q}$ such that $\mathrm{d} \vec{\rho}_{q}=\left(0,0, \ldots, \mathrm{d} \rho_{q}, \ldots, 0,0\right)$, and $\mathrm{d} \vec{\rho}_{q}$ implicitly depends on time. Finally, by substituting Eqs. (B14) and (B15) in Eq. (42), the inequality

$$
\frac{1}{\tilde{L}_{\mathrm{pw}}^{(q)}\left(\zeta_{1}, \vec{\rho}\right)} \frac{\partial \tilde{L}_{\mathrm{pw}}^{(q)}\left(\zeta_{1}, \vec{\rho}\right)}{\partial \rho_{q}}>\frac{1}{\tilde{L}_{\mathrm{pw}}^{(q)}\left(\zeta_{2}, \vec{\rho}\right)} \frac{\partial \tilde{L}_{\mathrm{pw}}^{(q)}\left(\zeta_{2}, \vec{\rho}\right)}{\partial \rho_{q}}
$$

is obtained. If the above inequality is satisfied, and if $\tilde{L}^{(q)}\left(\zeta_{k}, \vec{\rho}\right)$ [Eq. (B7)] is a continuous function, then a monotonic relationship between the TSD $\tilde{D}^{(q)}(\vec{\rho})$ and $\rho_{q}$ is achieved as a whole. We can easily prove that the absolute function in Eq. (B7) is continuous by using the $(\epsilon, \delta)$-definition of limit [17], and then $\tilde{L}^{(q)}\left(\zeta_{k}, \vec{\rho}\right)$ is also continuous. Note that the inner operation, the increment of $X_{\vec{\rho}}(t)$, of the absolute function is continuous with respect to $\vec{\rho}$ because $X_{\vec{\rho}}(t)$ changes with $\vec{\rho}$. This conditional equation is built on the appropriate values of $\Delta t, N$, and $\vec{\rho}$. Note that $\tilde{L}_{\mathrm{pw}}^{(q)}\left(\zeta_{1}, \vec{\rho}\right)$ and $\tilde{L}_{\mathrm{pw}}^{(q)}\left(\zeta_{2}, \vec{\rho}\right)$ can easily be obtained from Eq. (B14) by using $k=1$ and $k=2$, respectively. Likewise, $\partial \tilde{L}_{\mathrm{pw}}^{(q)}\left(\zeta_{1}, \vec{\rho}\right) / \partial \rho_{q}$ 
and $\partial \tilde{L}_{\mathrm{pw}}^{(q)}\left(\zeta_{2}, \vec{\rho}\right) / \partial \rho_{q}$ can be obtained from Eq. (B15) by considering $k=1$ and $k=2$, respectively.

The aforementioned analysis can be summarized in the following proposition.

Proposition B.1 (Monotonic relationship between the TSD and the noise level). Consider the d-dimensional linear stochastic system [Eq. (B1)]. Assume that only the variable $x_{q}$ is observed during the observational process and that observational noise does not influence the process. Then, if condition Eq. (B16) is satisfied, there exists a monotonic relationship between the $\operatorname{TSD} \tilde{D}^{(q)}(\vec{\rho})$ and the noise level $\rho_{q}(t) / \sigma_{\operatorname{dyn} x_{q}}(t)$.

The $\left(L_{\mathrm{seg}}(0, \Delta t), \rho_{q} / \sigma_{\operatorname{dyn} x_{q}}\right)$ diagram [see Fig. B1] shows the success rate in which the derived inequality [Eq. (B16)] holds given a noise level (uniformly distributed between 0.01 and 1) and length (uniformly distributed between 10 and 500 ) of a time series taken from the harmonic oscillator system [Eqs. (B3) and (B4)] simulated with the initial condition $x_{1}(0)=1$ and $x_{2}(0)=0$. The variable $x_{1}(t)$ was used as a target time series; namely, its curve length $\tilde{L}^{(1)}\left(\zeta_{k}, \vec{\rho}\right)$ was calculated. We calculated the inequality [Eq. (B16)] 100 times on each parameter point in the diagram to obtain the success rate. We define the success rate as the ratio between the number of successful simulations satisfying the inequality [Eq. (B16)] and the number of total simulations. In this simulation, $\rho_{1}$ and $\sigma_{\text {dyn } x_{1}}$ were constant for each trial. Then, the dynamical noise level of $x_{2}(t)$ was fixed at 0.1 for each trial. The sampling time $\Delta t$ was set to 0.001 . As a result, a larger size of data satisfies the inequality in a wider range of the noise level. Note that when the inequality is simulated, $\operatorname{Prob}\left[J_{\vec{\rho}}^{(q)}(m, j, k)=0\right]=0$ because $\vec{\rho}$ is optionally given, and thereby any used $\hat{\rho}_{q}$ is within $(S, E)$, that is $\hat{\rho}_{q} \in \Omega_{q}^{(S, E)}$ [see Eq. (40) and Eq. (41)]. Moreover, we have revealed that the original inequality [Eq. (43)] also shows the same diagram with Fig. B1, where $\tilde{D}_{\mathrm{pw}}^{(1)}\left(\rho_{1}+0.0001, \rho_{2}\right)-\tilde{D}_{\mathrm{pw}}^{(1)}\left(\rho_{1}, \rho_{2}\right)$ was used for calculation of the success rate. Then, the correlation coefficient between success rates of the original and the derived inequalities is more than 0.999. Thus, the derived inequality is valid for linear stochastic dynamical systems. 


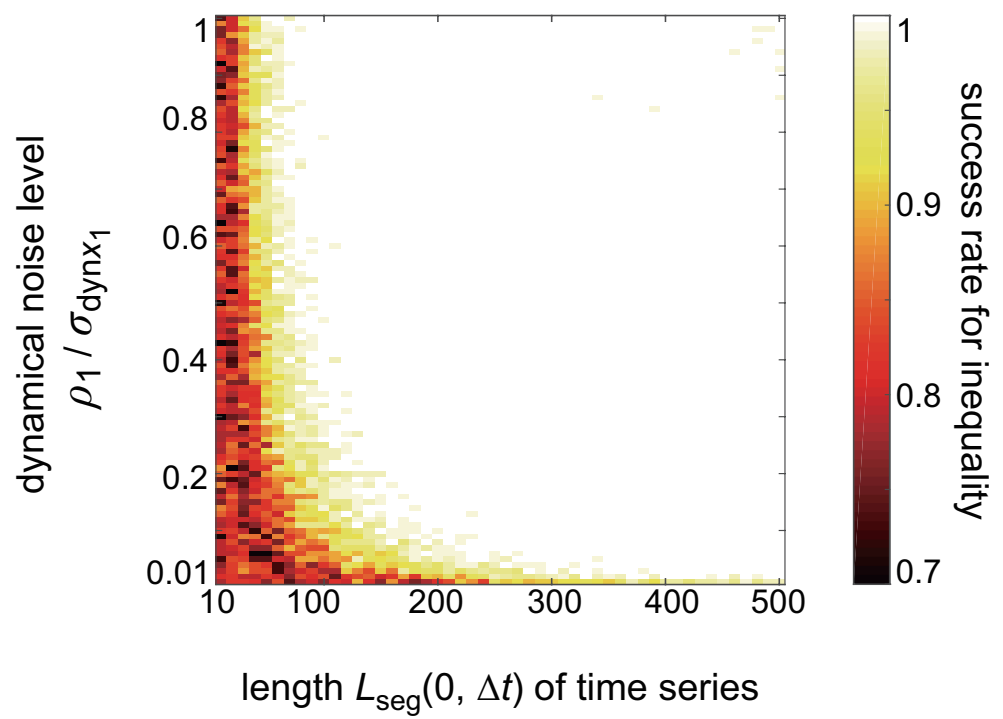

Figure B1: The $\left(L_{\mathrm{seg}}(0, \Delta t), \rho_{1} / \sigma_{\mathrm{dyn} x_{1}}\right)$ diagram in which the harmonic oscillator system was simulated. A larger success rate for the inequality is indicated by a lighter color.

\section{References}

[1] H. Kantz, T. Schreiber, Nonlinear Time Series Analysis, Cambridge University Press, Cambridge, MA, 1997, p. 174.

[2] M. R. Muldoon, D. S. Broomhead, J. P. Huke, R. Hegger, Dyn. Stab. Sys. $13(1998) 175$.

[3] A. Serletis, A. Shahmoradi, D. Serletis, Chaos Solitons Fractals 33 (2007) 914.

[4] J. P. M. Heald, J. Stark, Phys. Rev. Lett. 84 (2000) 2366.

[5] D. Orrell, Int. J. Bifurcat. Chaos 15 (2005) 99.

[6] M. Siefert, A. Kittel, R. Friedrich, J. Peinke, Europhys. Lett. 61 (2003) 466.

[7] A. N. Kolmogorov, Math. Ann. 140 (1931) 415. 
[8] J. E. Moyal, J. R. Stat. Soc. 11 (1949) 195.

[9] K. Urbanowicz, J. A. Holyst, Phys. Rev. E 67 (2003) 046218.

[10] P. Grassberger, I. Procaccia, Phys. Rev. A 28 (1983) 2591.

[11] L. G. G. Benettin, J. M. Strelcyn, Phys. Rev. A 14 (1976) 2338.

[12] J. L. Hindmarsh, R. M. Rose, Proc. R. Soc. London B 221 (1984) 87.

[13] D. Hansel, H. Sompolinsky, Phys. Rev. Lett. 68 (1992) 718.

[14] T. Higuchi, Physica D 31 (1998) 277.

[15] B. B. Mandelbrot, The Fractal Geometry of Nature, Freeman, San Francisco, 1982.

[16] T. Higuchi, Toukeisuri 37 (1989) 209.

[17] W. Rudin, Principles of Mathematical Analysis, McGraw-Hill, New York, 1967, p. 83.

[18] K. Kitajo, D. Nozaki, L. M. Ward, Y. Yamamoto, Phys. Rev. Lett. 90 (2003) 218103.

[19] K. Rektorys, Survey of Applicable Mathematics, Iliffe Books, London, 1969, p. 1125 . 